Published in final edited form as:

J Org Chem. 2018 March 16; 83(6): 3385-3391. doi:10.1021/acs.joc.8b00007.

\title{
Asymmetric Organocatalytic Sulfa-Michael Addition to Enone Diesters
}

\author{
Jennifer L. Fulton, Matthew A. Horwitz, Ericka L. Bruske, and Jeffrey S. Johnson * \\ Department of Chemistry, University of North Carolina at Chapel Hill, Chapel Hill, North Carolina \\ 27599-3290, United States
}

\begin{abstract}
An asymmetric sulfa-Michael addition of alkyl thiols to enone diesters is reported. The reaction is catalyzed by a bifunctional triaryliminophosphorane-thiourea organocatalyst and provides a range of $a$-sulfaketones in high yields and enantioselectivities. Leveraging the gem-diester functional handle via a subsequent diastereotopic group discrimination generates functionalized lactones with three contiguous stereocenters.
\end{abstract}

\section{Graphical Abstract}

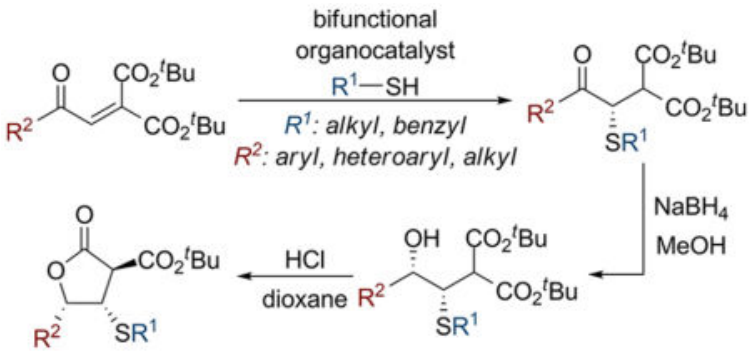

\begin{abstract}
The conjugate addition of heteroatom nucleophiles to prochiral Michael acceptors offers an atom-economical entry into diverse scaffolds with concomitant construction of stereochemical complexity. The application of this reaction manifold to the installation of sulfur in organic frameworks has largely been driven by sulfur's prominence in bioactive molecules ${ }^{1}$ and the established utility of thiol- and sulfide-derived functional handles. ${ }^{2}$ To that end, noteworthy advances have been made toward the development of the asymmetric sulfa-Michael reaction using a variety of catalytic systems. ${ }^{3}$ To advance the art, we sought to expand the range of products accessible through this reaction by studying substrates bearing multiple electrophilic sites on the Michael acceptor. Previous efforts in this direction have
\end{abstract}

\footnotetext{
*Corresponding Author: jsj@unc.edu. ORCID

Jeffrey S. Johnson: 0000-0001-8882-9881

Notes

The authors declare no competing financial interest.

Supporting Information

The Supporting Information is available free of charge on the ACS Publications website at DOI: 10.1021/acs.joc.8b00007.

Catalyst optimization and spectral data for all compounds (PDF)

Crystallographic data for $\mathbf{5 f}(\mathrm{CIF})$
} 
demonstrated that Michael acceptors possessing vicinal ester and ketone functionalities ( $\gamma$ oxo acrylates) give addition exclusively at the ester $a$-carbon, and achieving high stereoselectivities remains challenging (Scheme 1a). ${ }^{4}$ We viewed this shortcoming of cinchona alkaloid-based catalysts as an opportunity to explore organo-superbase catalysts for this transformation. Recently, the Dixon group developed a tert-leucine and phenylglycine-derived triaryliminophosphorane catalyst which promoted the enantioselective addition of alkyl thiols to unactivated $a, \beta$-unsaturated esters (Scheme 1b). ${ }^{5} \mathrm{In}$ addition, phenylglycine-based triaryliminophosphorane catalyst $\mathbf{C 1}$, also developed by the Dixon group, ${ }^{6}$ was found to mediate the addition of nitroalkanes to enone diesters with high regio- and stereoselectivity (Scheme 1c). ${ }^{7}$ By employing this catalyst system, we sought to overcome the challenges of a highly regio- and enantioselective addition of sulfur nucleophiles to acylidene malonates (Scheme 1d).

We began our studies by screening a number of catalysts (see the Supporting Information) and found that organocatalyst $\mathbf{C} \mathbf{1}$ chemoselectively added thiol 2a to acylidene malonate $\mathbf{1}$ to provide sulfide 3a in 97:3 er and 93\% yield (Table 1). With our optimized conditions in hand, we first examined the scope of the thiol nucleophile. We observed similar reaction outcomes with branched alkyl thiols (2b-c) as well as linear alkyl thiols (2d-e), although enantioselectivity was slightly diminished in the latter cases. Benzyl thiols also proved to be competent reaction partners, although it was important to use fewer equivalents of the thiol to maintain good yields and stereoselectivities (3f-3i) ${ }^{8}$ Employing benzyl thiol $\mathbf{2 f}$, the desired product (3f) was obtained in 91:9 er and $90 \%$ yield. Probing the impact of various para-substituents on the aromatic ring of the benzyl thiol, electron-withdrawing groups led to an increase in enantioselectivity (2g), while electron-donating groups had the opposite effect (2h-i).

Next, we investigated the scope of enone diesters that could be employed in this reaction (Table 2). Enone diesters bearing an electron-releasing acyl substituent (1j-k, 4-methoxy, 3methoxy, piperonyl) provided the desired products in excellent yield and enantioselectivity regardless of substitution pattern. Halogen-substituted arenes $(\mathbf{1 m}-\mathbf{0})$ were similarly successful under these reaction conditions, as were other electron-poor enone diesters (1pq). Cyclopropyl enone diester 1r provided the desired product (3r) in $96 \%$ yield, although the enantioselectivity was lowered to 83.5:16.5 er. An exploration of heteroaromatic enone diesters revealed that furan $\mathbf{3 s}$, thiophene $\mathbf{3 t}$, and pyridine $\mathbf{3 u}$ could all be obtained in good yield and enantioselectivity. When examining the scalability of this transformation, we observed that on a $1 \mathrm{~g}$ scale thiol 2a added to the acylidene malonate to afford $1.21 \mathrm{~g}$ of $a$ sulfaketone 3a without any negative impact on yield or stereoselectivity (Scheme 2).

Seeking to explore the synthetic utility of these products, we developed a two-step local desymmetrization that takes advantage of the diastereotopic ester groups (Scheme 3). ${ }^{9}$ Beginning with enantioenriched thioether 3a, reduction of the ketone with sodium borohydride ${ }^{10}$ provided the $s y n$-hydroxy sulfide $\mathbf{4 a}$ in $>20: 1 \mathrm{dr}$ and $89 \%$ yield. Subsequent acid- promoted cyclization provided lactone $\mathbf{5 a}$ in $>20: 1 \mathrm{dr}$ and $70 \%$ yield. Analogous results were obtained using benzyl thioether $\mathbf{3 f}$, with both the reduction and lactonization proceeding in good diastereoselectivity and yield. Finally, an X-ray diffraction study revealed the absolute and relative stereochemistry of lactone $\mathbf{5 f} .{ }^{11}$ 
In conclusion, we have developed an asymmetric organo-catalyzed addition of alkyl thiols to enone diesters with unique regioselectivity that enables access to an unexplored class of enantioenriched sulfa-Michael adducts. The diester products were further manipulated via diastereoselective reduction and subsequent diastereotopic group selection to form stereochemically dense lactone products.

\section{EXPERIMENTAL SECTION}

\section{General Comments}

Nuclear magnetic resonance spectra $\left({ }^{1} \mathrm{H}\right.$ NMR, ${ }^{13} \mathrm{C}$ NMR, ${ }^{19} \mathrm{~F}$ NMR $)$ were recorded at the following frequencies: ${ }^{1} \mathrm{H}$ NMR at 400 or $600 \mathrm{MHz},{ }^{13} \mathrm{C}$ NMR at 101 or $151 \mathrm{MHz},{ }^{19} \mathrm{~F}$ NMR at $376 \mathrm{MHz}$ with solvent resonance as the internal standard $\left({ }^{1} \mathrm{H} \mathrm{NMR}: \mathrm{CDCl}_{3}\right.$ at 7.26 ppm and ${ }^{13} \mathrm{C} \mathrm{NMR:} \mathrm{CDCl}_{3}$ at $\left.77.0 \mathrm{ppm}\right) .{ }^{1} \mathrm{H}$ NMR data are reported as follows: chemical shift, multiplicity ( $\mathrm{s}=$ singlet, $\mathrm{br} \mathrm{s}=$ broad singlet, $\mathrm{d}=$ doublet, $\mathrm{dd}=$ doublet of doublet, $\mathrm{t}=$ triplet, $\mathrm{m}=$ multiplet, app $\mathrm{t}=$ apparent triplet), coupling constants $(\mathrm{Hz})$, and integration.

High resolution mass spectra were obtained using a linear trap quadrupole Fourier transform (LTQ-FT) spectrometer. TLC visualization was accomplished using UV light, phosphomolybdic acid in ethanol, or aqueous ceric ammonium nitrate solution. HPLC analyses were carried out using Daicel Chiralpak IA, IC, and AD columns. Yields refer to isolated yields after flash column chromatography. Since all asymmetric trial results are the averages of two trials, the stereoisomer ratios listed in the paper may not exactly match those represented in the NMR and HPLC data below. Diethyl ether $\left(\mathrm{Et}_{2} \mathrm{O}\right)$ was passed through a column of neutral alumina under nitrogen prior to use. The thiols employed were obtained from commercial sources and used as received. Enone diesters were prepared according to a literature procedure. ${ }^{7}$ Triaryliminophosphorane catalysts $\mathbf{C 1}-\mathbf{C} 3$ were prepared according to a literature procedure. ${ }^{6 a}$ Racemic samples were obtained by employing the general procedure and using 2-tert-butylimino-2-diethylamino-1,3-dimethylperhydro-1,3,2diazaphosphorine (BEMP) in place of organocatalyst $\mathbf{C 1}$.

\section{General Procedure for the Asymmetric Conjugate Addition of Alkyl Thiols}

A flame-dried test tube was charged sequentially with enone diester 1 ( $0.1 \mathrm{mmol}, 1.0$ equiv) and $\mathrm{Et}_{2} \mathrm{O}(1.0 \mathrm{~mL})$. The reaction was stirred at $-60{ }^{\circ} \mathrm{C}$ in a cryogenic cooling apparatus for $15 \mathrm{~min}$, then triaryliminophosphorane catalyst $\mathbf{C 1}(0.01 \mathrm{mmol}, 0.10$ equiv) was added, followed by thiol 2 (see below for thiol identity and equivalents). The reaction was stirred at $-60{ }^{\circ} \mathrm{C}$ for $24 \mathrm{~h}$, then quenched with a TFA solution in toluene (50 $\mu \mathrm{L}, 0.5 \mathrm{M}$ solution) at the same temperature. The solvent was removed in vacuo, and the crude material was purified using flash column chromatography with 97.5/2.5 hexanes/EtOAc unless otherwise noted.

\section{Gram-Scale Asymmetric Conjugate Addition Reaction with Propane-2-thiol}

A flame-dried test tube was charged sequentially with enone diester $(1.00 \mathrm{~g}, 3.01 \mathrm{mmol}, 1.0$ equiv) and $\mathrm{Et}_{2} \mathrm{O}(30 \mathrm{~mL})$. The reaction was stirred at $-60{ }^{\circ} \mathrm{C}$ in a cryogenic cooling apparatus for $15 \mathrm{~min}$, then triaryliminophosphorane catalyst $\mathbf{C} 1$ (201 $\mathrm{mg}, 0.30 \mathrm{mmol}, 0.10$ equiv) was added, followed by propane-2-thiol ( $1.51 \mathrm{~mL}, 16.3 \mathrm{mmol}, 5.38$ equiv). The reaction was stirred at $-60^{\circ} \mathrm{C}$ for $24 \mathrm{~h}$, then quenched with a TFA solution in toluene (1.5 $\mathrm{mL}, 0.5 \mathrm{M}$ solution) at the same temperature. The solvent was removed in vacuo, and the 
crude material was purified using flash column chromatography with 97.5/2.5 hexanes/ EtOAc to yield $1.21 \mathrm{~g}(93 \%)$ of a low-melting white solid in 97:3 er.

Di-tert-butyl (S)-2-(1-(Isopropylthio)-2-oxo-2-phenylethyl)-malonate (3a)-The title compound was prepared using propane-2-thiol ( $0.05 \mathrm{~mL}, 0.54 \mathrm{mmol}, 5.38$ equiv) according to the general procedure. Low-melting white solid (38.3 mg, $0.093 \mathrm{mmol}, 93 \%$ ); ${ }^{1} \mathrm{H}$ NMR $\left(600 \mathrm{MHz}, \mathrm{CDCl}_{3}\right) \delta 8.05(\mathrm{~d}, J=7.9 \mathrm{~Hz}, 2 \mathrm{H}) ; 7.58(\mathrm{t}, J=7.3 \mathrm{~Hz}, 1 \mathrm{H}), 7.49(\mathrm{t}, J=$ $7.6 \mathrm{~Hz}, 2 \mathrm{H}), 4.76(\mathrm{~d}, J=11.6 \mathrm{~Hz}, 1 \mathrm{H}), 4.17(\mathrm{~d}, J=11.6 \mathrm{~Hz}, 1 \mathrm{H}), 2.96-2.89(\mathrm{~m}, 1 \mathrm{H}), 1.55$ (s, $9 \mathrm{H}), 1.39$ (s, 9H), $1.23(\mathrm{t}, J=6.8 \mathrm{~Hz}, 3 \mathrm{H}), 1.10(\mathrm{t}, J=6.8 \mathrm{~Hz}, 3 \mathrm{H}) ;{ }^{13} \mathrm{C} \mathrm{NMR}(151 \mathrm{MHz}$, $\left.\mathrm{CDCl}_{3}\right) \delta 194.8,167.0,166.9,135.9,133.0,128.7,128.6,82.4,82.2,55.8,45.7,34.6,28.0$, 27.8, 24.8, 24.2. IR (thin film) v3430, 2977, 2360, 1729, 1679, 1369, 1299, 1252, 1140, $690 \mathrm{~cm}^{-1}$. HRMS (ESI): Calcd For $\mathrm{C}_{22} \mathrm{H}_{32} \mathrm{O}_{5} \mathrm{SNa}^{+}\left(\left[\mathrm{M}+\mathrm{Na}^{+}\right]\right): 431.1868$, found 431.1858 . HPLC Chiralpak IC column, Hex $/{ }^{i} \mathrm{PrOH}=98: 2$, flow rate $=1.0 \mathrm{~mL} / \mathrm{min}, \lambda=210 \mathrm{~nm}, t_{\mathrm{R}}$ (minor) $11.5 \mathrm{~min}, t_{\mathrm{R}}$ (major) $15.4 \mathrm{~min}$. TLC (10/90 EtOAc/hexanes): $R_{f}=0.41$.

$[\alpha]_{\mathrm{D}}^{25}=-65.9\left(c=2.0, \mathrm{CHCl}_{3}\right)$.

Di-tert-butyl (S)-2-(1-(tert-Butylthio)-2-oxo-2-phenylethyl)-malonate (3b)-The title compound was prepared using 2-methylpropane-2-thiol $(0.06 \mathrm{~mL}, 0.53 \mathrm{mmol}, 5.32$ equiv) according to the general procedure. White solid (43.5 $\mathrm{mg}, 0.100 \mathrm{mmol}, 100 \%), \mathrm{mp}$ 76-78 ${ }^{\circ} \mathrm{C}$ (decomp); ${ }^{1} \mathrm{H}$ NMR (600 MHz, $\left.\mathrm{CDCl}_{3}\right) \delta 8.06(\mathrm{~d}, J=7.7 \mathrm{~Hz}, 2 \mathrm{H}), 7.56(\mathrm{t}, J=7.3$ $\mathrm{Hz}, 1 \mathrm{H}), 7.49$ (t, $J=7.6 \mathrm{~Hz}, 2 \mathrm{H}), 4.76(\mathrm{~d}, J=11.8 \mathrm{~Hz}, 1 \mathrm{H}), 4.17$ (d, $J=11.8 \mathrm{~Hz}, 1 \mathrm{H}), 1.55$ (s, 9H), 1.38 (s, 9H), 1.18 (s, 9H); $\left.{ }^{13} \mathrm{C} \mathrm{NMR} \mathrm{(151} \mathrm{MHz,} \mathrm{CDCl}_{3}\right) \delta 198.2,167.1$ (2C), 136.9, $132.7,129.1,128.4,82.5,82.1,57.0,45.6,45.4,31.5,28.0,27.8$. IR (thin film) $v 2979$, 2360, 1739, 1682, 1368, 1290, 1252, 1138, 855, $695 \mathrm{~cm}^{-1}$. HRMS (ESI): Calcd For $\mathrm{C}_{23} \mathrm{H}_{34} \mathrm{O}_{5} \mathrm{SNa}^{+}\left(\left[\mathrm{M}+\mathrm{Na}^{+}\right]\right): 445.2025$, found 445.2016. HPLC Chiralpak IC column, Hex/ ${ }^{i} \mathrm{PrOH}=96: 4$, flow rate $=1.0 \mathrm{~mL} / \mathrm{min}, \lambda=210 \mathrm{~nm}, t_{\mathrm{R}}$ (minor) $7.6 \mathrm{~min}, t_{\mathrm{R}}$ (major) $10.4 \mathrm{~min}$. $\operatorname{TLC}\left(10 / 90\right.$ EtOAc/hexanes): $R_{f}=0.42 .[\alpha]_{\mathrm{D}}^{25}=-89.2\left(c=2.0, \mathrm{CHCl}_{3}\right)$.

Di-tert-butyl (S)-2-(1-(Cyclohexylthio)-2-oxo-2-phenylethyl)-malonate (3c)-The title compound was prepared using cyclo-hexanethiol ( $0.06 \mathrm{~mL}, 0.49 \mathrm{mmol}, 4.90$ equiv) according to the general procedure. White solid ( $43.7 \mathrm{mg}, 0.097 \mathrm{mmol}, 97 \%), \mathrm{mp} 53-55{ }^{\circ} \mathrm{C}$ (decomp); ${ }^{1} \mathrm{H}$ NMR $\left(600 \mathrm{MHz}, \mathrm{CDCl}_{3}\right) \delta 8.04(\mathrm{~d}, J=7.9 \mathrm{~Hz}, 2 \mathrm{H}), 7.57(\mathrm{t}, J=7.3 \mathrm{~Hz}, 1 \mathrm{H})$, $7.49(\mathrm{t}, J=7.6 \mathrm{~Hz}, 2 \mathrm{H}), 4.76(\mathrm{~d}, J=11.6 \mathrm{~Hz}, 1 \mathrm{H}), 4.15(\mathrm{~d}, J=11.6 \mathrm{~Hz}, 1 \mathrm{H}), 2.71-2.67(\mathrm{~m}, 1$ H), 1.95-1.89 (m, 1H), 1.73-1.64 (m, 2H), 1.63-1.57 (m, 1H), $1.55(\mathrm{~s}, 9 \mathrm{H}), 1.53-1.48(\mathrm{~m}$, $1 \mathrm{H}), 1.38(\mathrm{~s}, 9 \mathrm{H}), 1.32-1.26(\mathrm{~m}, 2 \mathrm{H}), 1.25-1.19(\mathrm{~m}, 1 \mathrm{H}), 1.18-1.11(\mathrm{~m}, 2 \mathrm{H}) ;{ }^{13} \mathrm{C}$ NMR $(151$ $\left.\mathrm{MHz}, \mathrm{CDCl}_{3}\right) \delta 194.7,167.0,166.9,135.9,133.0,128.7,128.6,82.4,82.1,55.8,45.3,42.4$, $34.8,34.4,28.0,27.8,26.1,26.0,25.4$. IR (thin film) $v 2978,2931,2853,2360,1741,1680$, $1369,1299,1139,857 \mathrm{~cm}^{-1}$. HRMS (ESI): Calcd For $\mathrm{C}_{25} \mathrm{H}_{36} \mathrm{O}_{5} \mathrm{SNa}^{+}\left(\left[\mathrm{M}+\mathrm{Na}^{+}\right]\right)$: 471.2181, found 471.2171. HPLC Chiralpak IC column, $\mathrm{Hex} /{ }^{i} \mathrm{PrOH}=96: 4$, flow rate $=1.0$ $\mathrm{mL} / \mathrm{min}, \lambda=230 \mathrm{~nm}, t_{\mathrm{R}}$ (minor) $6.8 \mathrm{~min}, t_{\mathrm{R}}$ (major) $9.8 \mathrm{~min}$. TLC (10/90 EtOAc/ hexanes): $R_{f}=0.39 .[\alpha]_{\mathrm{D}}^{25}=-64.3\left(c=2.0, \mathrm{CHCl}_{3}\right)$.

Di-tert-butyl (S)-2-(1-(Ethylthio)-2-oxo-2-phenylethyl)malonate (3d)—The title compound was prepared using ethanethiol $(0.04 \mathrm{~mL}, 0.56 \mathrm{mmol}, 5.52$ equiv) according to 
the general procedure. Clear oil (36.5 mg, $0.092 \mathrm{mmol}, 92 \%) ;{ }^{1} \mathrm{H} \mathrm{NMR}\left(600 \mathrm{MHz}, \mathrm{CDCl}_{3}\right)$ $\delta 8.05(\mathrm{~d}, J=7.9 \mathrm{~Hz}, 2 \mathrm{H}), 7.58(\mathrm{t}, J=7.3 \mathrm{~Hz}, 1 \mathrm{H}), 7.50(\mathrm{t}, J=7.5 \mathrm{~Hz}), 4.73(\mathrm{~d}, J=11.5 \mathrm{~Hz}$, $1 \mathrm{H}), 4.14(\mathrm{~d}, J=11.5 \mathrm{~Hz}, 1 \mathrm{H}), 2.63-2.58(\mathrm{~m}, 1 \mathrm{H}), 2.39-2.33(\mathrm{~m}, 1 \mathrm{H}), 1.55(\mathrm{~s}, 9 \mathrm{H}), 1.39$ (s, $9 \mathrm{H}), 1.14(\mathrm{t}, J=7.5 \mathrm{~Hz}, 3 \mathrm{H}) ;{ }^{13} \mathrm{C} \mathrm{NMR}\left(151 \mathrm{MHz}, \mathrm{CDCl}_{3}\right) \delta 193.1,167.0,166.8,135.5$, 133.1, 128.7, 128.6, 82.5, 82.2, 54.8, 44.9, 28.0, 27.8, 22.9, 13.8. IR (thin film) $v 3431$, 2978, 1741, 1679, 1369, 1300, 1259, 1140, 856, $689 \mathrm{~cm}^{-1}$. HRMS (ESI): Calcd For $\mathrm{C}_{21} \mathrm{H}_{30} \mathrm{O}_{5} \mathrm{SNa}^{+}\left(\left[\mathrm{M}+\mathrm{Na}^{+}\right]\right): 417.1708$, found 417.1701. HPLC Chiralpak IC column, Hex/ ${ }^{i} \mathrm{PrOH}=98: 2$, flow rate $=1.0 \mathrm{~mL} / \mathrm{min}, \lambda=210 \mathrm{~nm}, t_{\mathrm{R}}$ (minor) $9.5 \mathrm{~min}, t_{\mathrm{R}}$ (major) $14.8 \mathrm{~min}$. $\operatorname{TLC}\left(10 / 90\right.$ EtOAc/hexanes): $R_{f}=0.39 .[\alpha]_{\mathrm{D}}^{25}=-44.2\left(c=2.0, \mathrm{CHCl}_{3}\right)$.

Di-tert-butyl (S)-2-(2-Oxo-2-phenyl-1-(propylthio)ethyl)malonate (3e)-The title compound was prepared using propane-1-thiol $(0.05 \mathrm{~mL}, 0.54 \mathrm{mmol}, 5.36$ equiv) according to the general procedure. Clear oil $(39.5 \mathrm{mg}, 0.096 \mathrm{mmol}, 96 \%) ;{ }^{1} \mathrm{H} \mathrm{NMR}(600 \mathrm{MHz}$, $\left.\mathrm{CDCl}_{3}\right) \delta 8.05(\mathrm{~d}, J=7.7 \mathrm{~Hz}, 2 \mathrm{H}), 7.58(\mathrm{t}, J=7.3 \mathrm{~Hz}, 1 \mathrm{H}), 7.49(\mathrm{t}, J=7.6 \mathrm{~Hz}, 2 \mathrm{H}), 4.73(\mathrm{~d}$, $J=11.5 \mathrm{~Hz}, 1 \mathrm{H}), 4.13(\mathrm{~d}, J=11.5 \mathrm{~Hz}, 1 \mathrm{H}), 2.58-2.53(\mathrm{~m}, 1 \mathrm{H}), 2.33-2.29(\mathrm{~m}, 1 \mathrm{H}), 1.55(\mathrm{~s}$, 9H), $1.52-1.46$ (m, 2H), 1.39 (s, 9H), 0.90 (t, $J=7.4 \mathrm{~Hz}, 3 \mathrm{H}) ;{ }^{13} \mathrm{C}$ NMR $\left(151 \mathrm{MHz}, \mathrm{CDCl}_{3}\right)$ $\delta 193.2,166.9,166.8,135.5,133.0,128.7,128.6,82.4,82.1,54.8,44.7,30.6,28.0,27.8$, 22.3, 13.6. IR (thin film) $v 3431,2978,2360,1740,1680,1369,1299,1139,856,668 \mathrm{~cm}^{-1}$. HRMS (ESI): Calcd For $\mathrm{C}_{22} \mathrm{H}_{32} \mathrm{O}_{5} \mathrm{SNa}^{+}\left(\left[\mathrm{M}+\mathrm{Na}^{+}\right]\right): 431.1868$, found 431.1858. HPLC Chiralpak IC column, $\mathrm{Hex} /{ }^{i} \mathrm{PrOH}=98: 2$, flow rate $=1.0 \mathrm{~mL} / \mathrm{min}, \lambda=210 \mathrm{~nm}, t_{\mathrm{R}}($ minor $)$ $6.6 \mathrm{~min}, t_{\mathrm{R}}$ (major) $9.4 \mathrm{~min}$. TLC (10/90 EtOAc/ hexanes): $R_{f}=0.41 .[\alpha]_{\mathrm{D}}^{25}=-54.0(c=2.0$, $\left.\mathrm{CHCl}_{3}\right)$.

Di-tert-butyl (S)-2-(1-(Benzylthio)-2-oxo-2-phenylethyl)malonate (3f)-The title compound was prepared using phenylmethanethiol $(0.02 \mathrm{~mL}, 0.17 \mathrm{mmol}, 1.71$ equiv) according to the general procedure. Low-melting white solid (40.4 mg, $0.091 \mathrm{mmol}, 91 \%$ ); ${ }^{1} \mathrm{H}$ NMR $\left(600 \mathrm{MHz}, \mathrm{CDCl}_{3}\right) \delta 7.98(\mathrm{~d}, J=7.9 \mathrm{~Hz}, 2 \mathrm{H}), 7.58(\mathrm{t}, J=7.4 \mathrm{~Hz}, 1 \mathrm{H}), 7.47(\mathrm{t}, J=$ $7.6 \mathrm{~Hz}, 2 \mathrm{H}), 7.29-7.22(\mathrm{~m}, 5 \mathrm{H}), 4.79(\mathrm{~d}, J=11.4 \mathrm{~Hz}, 1 \mathrm{H}), 4.26$ (d, $J=11.4 \mathrm{~Hz}, 1 \mathrm{H}), 3.86$ $(\mathrm{d}, J=11.8 \mathrm{~Hz}, 1 \mathrm{H}), 3.55(\mathrm{~d}, J=11.8 \mathrm{~Hz}, 1 \mathrm{H}), 1.58(\mathrm{~s}, 9 \mathrm{H}), 1.40(\mathrm{~s}, 9 \mathrm{H}) ;{ }^{13} \mathrm{C}$ NMR $(151$ $\mathrm{MHz}, \mathrm{CDCl}_{3}$ ) $\delta 193.2,166.9$ (2C), 136.2, 135.4, 133.1, 129.4, 128.7, 128.6, 128.5, 127.3, 82.6, 82.3, 55.0, 45.3, 33.7, 28.0, 27.8. IR (thin film) v 3431, 2978, 2360, 1727, 1678, 1369, $1300,1252,1157,691 \mathrm{~cm}^{-1}$. HRMS (ESI): Calcd For $\mathrm{C}_{26} \mathrm{H}_{32} \mathrm{O}_{5} \mathrm{SNa}^{+}\left(\left[\mathrm{M}+\mathrm{Na}^{+}\right]\right)$: 479.1868, found 479.1858. HPLC Chiralpak IC column, $\mathrm{Hex} / \mathrm{i} \mathrm{PrOH}=99: 1$, flow rate $=1.0$ $\mathrm{mL} / \mathrm{min}, \lambda=210 \mathrm{~nm}, t_{\mathrm{R}}$ (major) $10.8 \mathrm{~min}, t_{\mathrm{R}}$ (minor) $12.3 \mathrm{~min}$. TLC (10/90 EtOAc/ hexanes): $R_{f}=0.36 .[a]_{\mathrm{D}}^{25}=-50.6\left(c=2.0, \mathrm{CHCl}_{3}\right)$.

\section{Di-tert-butyl (S)-2-(1-((4-Chlorobenzyl)thio)-2-oxo-2-phenylethyl)malonate (3g)}

-The title compound was prepared using (4-chlorophenyl)methanethiol $(0.02 \mathrm{~mL}, 0.15$ mmol, 1.51 equiv) according to the general procedure. Low-melting white solid (44.4 $\mathrm{mg}$, $0.089 \mathrm{mmol}, 89 \%) ;{ }^{1} \mathrm{H}$ NMR $\left(600 \mathrm{MHz}, \mathrm{CDCl}_{3}\right) \delta 7.94(\mathrm{~d}, J=7.9 \mathrm{~Hz}, 2 \mathrm{H}), 7.58(\mathrm{t}, J=7.4$ $\mathrm{Hz}, 1 \mathrm{H}), 7.47$ (t, $J=7.7 \mathrm{~Hz}, 2 \mathrm{H}), 7.21(\mathrm{~d}, J=8.3 \mathrm{~Hz}, 2 \mathrm{H}), 7.15(\mathrm{~d}, J=8.2 \mathrm{~Hz}, 2 \mathrm{H}), 4.76(\mathrm{~d}$, $J=11.5 \mathrm{~Hz}, 1 \mathrm{H}), 4.23(\mathrm{~d}, J=11.5 \mathrm{~Hz}, 1 \mathrm{H}), 3.81(\mathrm{~d}, J=12.1 \mathrm{~Hz}, 1 \mathrm{H}), 3.51(\mathrm{~d}, J=12.1 \mathrm{~Hz}$, 1H), 1.57 (s, 9H), 1.38 (s, 9H); ${ }^{13} \mathrm{C} \mathrm{NMR}\left(151 \mathrm{MHz}, \mathrm{CDCl}_{3}\right) \delta 193.2,166.8(2 \mathrm{C}), 135.3$, 134.9, 133.2, 133.1, 130.7, 128.7, 128.6 (2C), 82.7, 82.4, 55.0, 45.1, 33.1, 28.0, 27.8. IR (thin film) $v 3431,2979,2360,1739,1678,1491,1369,1301,1140,690 \mathrm{~cm}^{-1}$. HRMS 
(ESI): Calcd For $\mathrm{C}_{26} \mathrm{H}_{31} \mathrm{ClO}_{5} \mathrm{SNa}^{+}$([M + $\left.\left.\mathrm{Na}^{+}\right]\right): 513.1479$, found 513.1469. HPLC Chiralpak IC column, $\mathrm{Hex} / \mathrm{i} \mathrm{PrOH}=98: 2$, flow rate $=1.0 \mathrm{~mL} / \mathrm{min}, \lambda=210 \mathrm{~nm}, t_{\mathrm{R}}$ (major)

$9.1 \mathrm{~min}, t_{\mathrm{R}}$ (minor) $12.2 \mathrm{~min}$. TLC (10/90 EtOAc/hexanes): $R_{f}=0.41 .[\alpha]_{\mathrm{D}}^{25}=-54.6(c=2.0$, $\left.\mathrm{CHCl}_{3}\right)$.

\section{Di-tert-butyl (S)-2-(1-((4-Methoxybenzyl)thio)-2-oxo-2-phenylethyl)malonate}

(3h) - The title compound was prepared using (4-methoxyphenyl)methanethiol $(0.03 \mathrm{~mL}$, $0.21 \mathrm{mmol}, 2.15$ equiv) according to the general procedure. The crude material was purified via flash column chromatography using a gradient from 97.5/ 2.5 hexanes/EtOAc to 95/5 hexanes/EtOAc. Low-melting white solid (45.2 mg, $0.094 \mathrm{mmol}, 94 \%) ;{ }^{1} \mathrm{H}$ NMR (600 $\left.\mathrm{MHz}, \mathrm{CDCl}_{3}\right) \delta 7.97(\mathrm{~d}, J=7.9 \mathrm{~Hz}, 2 \mathrm{H}), 7.58(\mathrm{t}, J=7.3 \mathrm{~Hz}, 1 \mathrm{H}), 7.46(\mathrm{t}, J=7.6 \mathrm{~Hz}, 2 \mathrm{H})$, $7.14(\mathrm{~d}, J=8.4 \mathrm{~Hz}, 2 \mathrm{H}), 6.79(\mathrm{~d}, J=8.4 \mathrm{~Hz}, 2 \mathrm{H}), 4.76(\mathrm{~d}, J=11.5 \mathrm{~Hz}, 1 \mathrm{H}), 4.26(\mathrm{~d}, J=11.5$ $\mathrm{Hz}, 1 \mathrm{H}), 3.82(\mathrm{~d}, J=11.8 \mathrm{~Hz}, 1 \mathrm{H}), 3.78(\mathrm{~s}, 3 \mathrm{H}), 3.50(\mathrm{~d}, J=11.8 \mathrm{~Hz}, 1 \mathrm{H}), 1.58(\mathrm{~s}, 9 \mathrm{H}), 1.40$ (s, 9H); ${ }^{13} \mathrm{C}$ NMR $\left(151 \mathrm{MHz}, \mathrm{CDCl}_{3}\right) \delta 193.2,166.9$ (2C), 158.8, 135.4, 133.0, 130.5, 128.7, 128.6, 128.0, 113.9, 82.5, 82.3, 55.2, 54.5, 45.3, 33.2, 28.0, 27.8. IR (thin film) $v$ 3431, 2978, 2360, 1777, 1738, 1512, 1251, 1139, 833, $690 \mathrm{~cm}^{-1}$. HRMS (ESI): Calcd For $\mathrm{C}_{27} \mathrm{H}_{34} \mathrm{O}_{6} \mathrm{SNa}^{+}\left(\left[\mathrm{M}+\mathrm{Na}^{+}\right]\right): 509.1974$, found 509.1967. HPLC Chiralpak IC column, Hex/ ${ }^{i} \mathrm{PrOH}=98: 2$, flow rate $=1.0 \mathrm{~mL} / \mathrm{min}, \lambda=210 \mathrm{~nm}, t_{\mathrm{R}}$ (major) $14.8 \mathrm{~min}, t_{\mathrm{R}}$ (minor) 18.1 $\min$. TLC (10/90 EtOAc/hexanes): $R_{f}=0.25 .[\alpha]_{\mathrm{D}}^{25}=-49.9\left(c=2.0, \mathrm{CHCl}_{3}\right)$.

\section{Di-tert-butyl (S)-2-(1-((4-(tert-Butyl)benzyl)thio)-2-oxo-2-phenylethyl)malonate}

(3i) - The title compound was prepared using (4-tert-butylphenyl)methanethiol $(0.03 \mathrm{~mL}$, $0.16 \mathrm{mmol}, 1.60$ equiv) according to the general procedure. Clear oil ( $44.4 \mathrm{mg}, 0.086 \mathrm{mmol}$, $86 \%) ;{ }^{1} \mathrm{H}$ NMR $\left(600 \mathrm{MHz}, \mathrm{CDCl}_{3}\right) \delta 7.98(\mathrm{~d}, J=7.8 \mathrm{~Hz}, 2 \mathrm{H}), 7.58(\mathrm{t}, J=7.4 \mathrm{~Hz}, 1 \mathrm{H}), 7.47$ $(\mathrm{t}, J=7.5 \mathrm{~Hz}, 2 \mathrm{H}), 7.28(\mathrm{~d}, J=7.9 \mathrm{~Hz}, 2 \mathrm{H}), 7.15(\mathrm{~d}, J=7.9 \mathrm{~Hz}, 2 \mathrm{H}), 4.79(\mathrm{~d}, J=11.4 \mathrm{~Hz}$, $1 \mathrm{H}), 4.26(\mathrm{~d}, J=11.5 \mathrm{~Hz}, 1 \mathrm{H}), 3.86(\mathrm{~d}, J=11.8 \mathrm{~Hz}, 1 \mathrm{H}), 3.53(\mathrm{~d}, J=11.7 \mathrm{~Hz}, 1 \mathrm{H}), 1.59$ (s, 9H), $1.41(\mathrm{~s}, 9 \mathrm{H}), 1.30(\mathrm{~s}, 9 \mathrm{H}) ;{ }^{13} \mathrm{C}$ NMR $\left(151 \mathrm{MHz}, \mathrm{CDCl}_{3}\right) \delta$ 193.2, $166.9(2 \mathrm{C}), 150.2$, 135.5, 133.0 (2C), 129.0, 128.7, 128.6, 125.4, 82.5, 82.3, 54.9, 45.4, 34.5, 33.2, 31.3, 28.0, 27.8. IR (thin film) $v 3431,2970,2360,1728,1678,1368,1300,1253,1139,851 \mathrm{~cm}^{-1}$. HRMS (ESI): Calcd For $\mathrm{C}_{30} \mathrm{H}_{40} \mathrm{O}_{5} \mathrm{SNa}^{+}\left(\left[\mathrm{M}+\mathrm{Na}^{+}\right]\right)$: 535.2494, found 535.2491. HPLC Chiralpak AD column, $\mathrm{Hex} /{ }^{i} \mathrm{PrOH}=99: 1$, flow rate $=1.0 \mathrm{~mL} / \mathrm{min}, \lambda=254 \mathrm{~nm}, t_{\mathrm{R}}$ (minor) $7.7 \mathrm{~min}, t_{\mathrm{R}}$ (major) $9.5 \mathrm{~min}$. TLC (10/90 EtOAc/hexanes): $R_{f}=0.39 .[\alpha]_{\mathrm{D}}^{25}=-48.3(c=2.0$, $\left.\mathrm{CHCl}_{3}\right)$.

\section{Di-tert-butyl (S)-2-(1-(Isopropylthio)-2-(4-methoxyphenyl)-2-oxoethyl)malonate}

(3j) - The title compound was prepared according to the general procedure using propane-2thiol $(0.05 \mathrm{~mL}, 0.54 \mathrm{mmol}, 5.38$ equiv). The crude material was purified via flash column chromatography using a gradient from $97.5 / 2.5$ hexanes/EtOAc to $95 / 5$ hexanes/EtOAc. Clear oil (43.6 mg, $0.099 \mathrm{mmol}, 99 \%) ;{ }^{1} \mathrm{H}$ NMR $\left(600 \mathrm{MHz}, \mathrm{CDCl}_{3}\right) \delta 8.04(\mathrm{~d}, J=8.9 \mathrm{~Hz}$, 2H), $6.97(\mathrm{~d}, J=8.9 \mathrm{~Hz}, 2 \mathrm{H}), 4.73(\mathrm{~d}, J=11.6 \mathrm{~Hz}, 1 \mathrm{H}), 4.16(\mathrm{~d}, J=11.5 \mathrm{~Hz}, 1 \mathrm{H}), 3.89$ (s, $3 \mathrm{H}), 2.97-2.90(\mathrm{~m}, 1 \mathrm{H}), 1.55$ (s, 9H), $1.38(\mathrm{~s}, 9 \mathrm{H}), 1.24(\mathrm{~d}, J=6.8 \mathrm{~Hz}, 3 \mathrm{H}), 1.10$ (d, $J=6.8$ $\mathrm{Hz}, 3 \mathrm{H}) ;{ }^{13} \mathrm{C}$ NMR $\left(151 \mathrm{MHz}, \mathrm{CDCl}_{3}\right) \delta 193.5,167.1,167.0,163.5,131.0,128.6,113.8$, 82.3, 82.1, 55.8, 55.5, 45.3, 34.4, 28.0, 27.8, 24.8, 24.2. IR (thin film) v3431, 2978, 2360, $1739,1669,1602,1369,1260,1142,860 \mathrm{~cm}^{-1}$. HRMS (ESI): Calcd For $\mathrm{C}_{23} \mathrm{H}_{34} \mathrm{O}_{6} \mathrm{SNa}^{+}$ 
$\left(\left[\mathrm{M}+\mathrm{Na}^{+}\right]\right): 461.1974$, found 461.1964. HPLC Chiralpak IA column, Hex $/{ }^{i} \mathrm{PrOH}=98: 2$, flow rate $=1.0 \mathrm{~mL} / \mathrm{min}, \lambda=210 \mathrm{~nm}, t_{\mathrm{R}}$ (major) $9.8 \mathrm{~min}, t_{\mathrm{R}}$ (minor) $10.7 \mathrm{~min}$. TLC (10/90 EtOAc/hexanes): $R_{f}=0.29 .[\alpha]_{\mathrm{D}}^{25}=-43.7\left(c=2.0, \mathrm{CHCl}_{3}\right)$.

\section{Di-tert-butyl (S)-2-(1-(Isopropylthio)-2-(3-methoxyphenyl)-2-oxoethyl)malonate}

(3k)-The title compound was prepared according to the general procedure using propane-2-thiol ( $0.05 \mathrm{~mL}, 0.54 \mathrm{mmol}, 5.38$ equiv). The crude material was purified via flash column chromatography using a gradient from 97.5/2.5 hexanes/EtOAc to 95/5 hexanes/ EtOAc. Clear oil (42.3 mg, $0.096 \mathrm{mmol}, 96 \%) ;{ }^{1} \mathrm{H}$ NMR (600 MHz, $\left.\mathrm{CDCl}_{3}\right) \delta 7.64$ (d, $J=$ $7.7 \mathrm{~Hz}, 1 \mathrm{H}), 7.56(\mathrm{dd}, J=2.4 \mathrm{~Hz}, 1.6 \mathrm{~Hz}, 1 \mathrm{H}), 7.40(\mathrm{t}, J=8.0 \mathrm{~Hz}, 1 \mathrm{H}), 7.12(\mathrm{dd}, J=8.2 \mathrm{~Hz}$, $2.6 \mathrm{~Hz}, 1 \mathrm{H}), 4.73(\mathrm{~d}, J=11.6 \mathrm{~Hz}, 1 \mathrm{H}), 4.16(\mathrm{~d}, J=11.6 \mathrm{~Hz}, 1 \mathrm{H}), 2.96-2.89(\mathrm{~m}, 1 \mathrm{H}), 1.55$ (s, 9H), 1.39 (s, 9H), 1.23 (d, $J=6.8 \mathrm{~Hz}, 3 \mathrm{H}), 1.12(\mathrm{~d}, J=6.8 \mathrm{~Hz}, 3 \mathrm{H}) ;{ }^{13} \mathrm{C}$ NMR $(151 \mathrm{MHz}$, $\left.\mathrm{CDCl}_{3}\right) \delta 194.6,166.9(2 \mathrm{C}), 159.8,137.3,129.6,121.2,119.5,113.1,82.5,82.2,55.8,55.4$, $45.7,34.7,28.0,27.8,24.7,24.2$. IR (thin film) $v 3432,2978,2360,1729,1679,1583$, $1369,1288,1159,761 \mathrm{~cm}^{-1}$. HRMS (ESI): Calcd For $\mathrm{C}_{23} \mathrm{H}_{34} \mathrm{O}_{6} \mathrm{SNa}^{+}\left(\left[\mathrm{M}+\mathrm{Na}^{+}\right]\right)$: 461.1974, found 461.1963. HPLC Chiralpak IA column, $\mathrm{Hex} /{ }^{i} \mathrm{PrOH}=96: 4$, flow rate $=1.0$ $\mathrm{mL} / \mathrm{min}, \lambda=210 \mathrm{~nm}, t_{\mathrm{R}}$ (minor) $8.2 \mathrm{~min}, t_{\mathrm{R}}$ (major) $12.7 \mathrm{~min}$. TLC (10/90 EtOAc/hexanes): $R_{f}=0.35 .[\alpha]_{\mathrm{D}}^{25}=-74.5\left(c=2.0, \mathrm{CHCl}_{3}\right)$.

\section{Di-tert-butyl (S)-2-(2-(Benzo[d][1,3]dioxol-5-yl)-1-(isopropylthio)-2-} oxoethyl)malonate (3I)-The title compound was prepared according to the general procedure using propane-2-thiol ( $0.05 \mathrm{~mL}, 0.54 \mathrm{mmol}, 5.38$ equiv). The crude material was purified via flash column chromatography using a gradient from 97.5/2.5 hexanes/ EtOAc to 95/5 hexanes/EtOAc. Clear oil (42.3 mg, $0.093 \mathrm{mmol}, 93 \%) ;{ }^{1} \mathrm{H} \mathrm{NMR}\left(600 \mathrm{MHz}, \mathrm{CDCl}_{3}\right) \delta$ $7.68(\mathrm{dd}, J=8.2 \mathrm{~Hz}, 1.7 \mathrm{~Hz}, 1 \mathrm{H}), 7.51(\mathrm{~d}, J=1.7 \mathrm{~Hz}, 1 \mathrm{H}), 6.89(\mathrm{~d}, J=8.2 \mathrm{~Hz}, 1 \mathrm{H}), 6.06(\mathrm{~s}$, $2 \mathrm{H}), 4.67(\mathrm{~d}, J=11.6 \mathrm{~Hz}, 1 \mathrm{H}), 4.14(\mathrm{~d}, J=11.5 \mathrm{~Hz}, 1 \mathrm{H}), 2.97-2.90(\mathrm{~m}, 1 \mathrm{H}), 1.54(\mathrm{~s}, 9 \mathrm{H})$, $1.38(\mathrm{~s}, 9 \mathrm{H}), 1.24$ (d, $J=6.9 \mathrm{~Hz}, 3 \mathrm{H}), 1.11(\mathrm{~d}, J=6.8 \mathrm{~Hz}, 3 \mathrm{H}) ;{ }^{13} \mathrm{C} \mathrm{NMR}(151 \mathrm{MHz}$, $\left.\mathrm{CDCl}_{3}\right) \delta 193.0,167.0,166.9,151.8,148.1,130.4,124.8,108.7,108.0,101.9,82.4,82.1$, 55.9, 45.4, 34.5, 28.0, 27.8, 24.8, 24.2. IR (thin film) $v 3431,2978,1727,1672,1442,1258$, 1139, 1038, 850, $734 \mathrm{~cm}^{-1}$. HRMS (ESI): Calcd For $\mathrm{C}_{23} \mathrm{H}_{33} \mathrm{O}_{7} \mathrm{~S}^{+}\left(\left[\mathrm{M}+\mathrm{H}^{+}\right]\right): 453.1948$, found 453.1940. HPLC Chiralpak IA column, $\mathrm{Hex} / \mathrm{PrOH}=95: 5$, flow rate $=1.0 \mathrm{~mL} / \mathrm{min}, \lambda$ $=210 \mathrm{~nm}, t_{\mathrm{R}}$ (major) $5.5 \mathrm{~min}, t_{\mathrm{R}}$ (minor) $6.2 \mathrm{~min}$. TLC (10/90 EtOAc/hexanes): $R_{f}=0.30$.

$[\alpha]_{\mathrm{D}}^{25}=-51.8\left(c=2.0, \mathrm{CHCl}_{3}\right)$.

\section{Di-tert-butyl (S)-2-(2-(4-Bromophenyl)-1-(isopropylthio)-2-oxoethyl)malonate}

$(3 \mathrm{~m})$-The title compound was prepared according to the general procedure using propane-2-thiol ( $0.05 \mathrm{~mL}, 0.54 \mathrm{mmol}, 5.38$ equiv). White solid $(46.8 \mathrm{mg}, 0.096 \mathrm{mmol}$, 96\%), mp 73-75 ${ }^{\circ} \mathrm{C}$ (decomp); ${ }^{1} \mathrm{H} \mathrm{NMR}\left(600 \mathrm{MHz}, \mathrm{CDCl}_{3}\right) \delta 7.92(\mathrm{~d}, J=8.6 \mathrm{~Hz}, 2 \mathrm{H}), 7.64$ (d, $J=8.6 \mathrm{~Hz}, 2 \mathrm{H}), 4.69(\mathrm{~d}, J=11.6 \mathrm{~Hz}, 1 \mathrm{H}), 4.15(\mathrm{~d}, J=11.6 \mathrm{~Hz}, 1 \mathrm{H}), 2.94-2.87(\mathrm{~m}, 1 \mathrm{H})$, 1.55 (s, 9H), 1.39 (s, 9H), 1.24 (d, $J=6.8 \mathrm{~Hz}, 3 \mathrm{H}), 1.09$ (d, $J=6.8 \mathrm{~Hz}, 3 \mathrm{H}) ;{ }^{13} \mathrm{C}$ NMR $(151$ $\left.\mathrm{MHz}, \mathrm{CDCl}_{3}\right) \delta 193.8,167.0,166.8,134.7,131.9,130.2,128.1,82.6,82.3,55.7,45.7,34.7$, 28.0, 27.8, 24.8, 24.2. IR (thin film) v 3430, 2978, 2360, 1729, 1679, 1586, 1369, 1309, 1251, $1139 \mathrm{~cm}^{-1}$. HRMS (ESI): Calcd For $\mathrm{C}_{22} \mathrm{H}_{31} \mathrm{BrO}_{5} \mathrm{SNa}^{+}\left(\left[\mathrm{M}+\mathrm{Na}^{+}\right]\right): 509.0974$, found 509.0963. HPLC Chiralpak IC column, $\mathrm{Hex} /{ }^{i} \mathrm{PrOH}=98: 2$, flow rate $=1.0 \mathrm{~mL} / \mathrm{min}, \lambda=210$ 
$\mathrm{nm}, t_{\mathrm{R}}$ (minor) $5.8 \mathrm{~min}, t_{\mathrm{R}}$ (major) $8.0 \mathrm{~min}$. TLC (10/90 EtOAc/hexanes): $R_{f}=0.50$.

$[\alpha]_{\mathrm{D}}^{25}=-41.3\left(c=2.0, \mathrm{CHCl}_{3}\right)$.

\section{Di-tert-butyl (S)-2-(2-(4-Chlorophenyl)-1-(isopropylthio)-2-oxoethyl)malonate}

$(3 n)-T h e$ title compound was prepared according to the general procedure using propane-2-thiol $(0.05 \mathrm{~mL}, 0.54 \mathrm{mmol}, 5.38$ equiv). White solid $(43.6 \mathrm{mg}, 0.098 \mathrm{mmol}$, 98\%), mp 64-66 ${ }^{\circ} \mathrm{C}$ (decomp); ${ }^{1} \mathrm{H}$ NMR (600 MHz, $\left.\mathrm{CDCl}_{3}\right) \delta 7.99(\mathrm{~d}, J=8.6 \mathrm{~Hz}, 2 \mathrm{H}), 7.47$ (d, $J=8.6 \mathrm{~Hz}, 2 \mathrm{H}), 4.69$ (d, $J=11.6 \mathrm{~Hz}, 1 \mathrm{H}), 4.15(\mathrm{~d}, J=11.6 \mathrm{~Hz}, 1 \mathrm{H}), 2.94-2.87(\mathrm{~m}, 1 \mathrm{H})$, 1.55 (s, 9H), 1.39 (s, 9H), $1.24(\mathrm{~d}, J=6.8 \mathrm{~Hz}, 3 \mathrm{H}), 1.09$ (d, $J=6.8 \mathrm{~Hz}, 3 \mathrm{H}) ;{ }^{13} \mathrm{C}$ NMR $(151$ $\left.\mathrm{MHz}, \mathrm{CDCl}_{3}\right) \delta 193.6,167.0,166.8,139.4,134.2,130.1,128.9,82.6,82.3,55.7,45.7,34.6$, 27.9, 27.8, 24.8, 24.2. IR (thin film) v 3430, 2979, 1740, 1680, 1590, 1369, 1309, 1140, 1093, $861 \mathrm{~cm}^{-1}$. HRMS (ESI): Calcd For $\mathrm{C}_{22} \mathrm{H}_{31} \mathrm{ClO}_{5} \mathrm{SNa}^{+}\left(\left[\mathrm{M}+\mathrm{Na}^{+}\right]\right): 465.1479$, found 465.1469. HPLC Chiralpak IC column, $\mathrm{Hex} /{ }^{i} \mathrm{PrOH}=98: 2$, flow rate $=1.0 \mathrm{~mL} / \mathrm{min}, \lambda=210$ $\mathrm{nm}, t_{\mathrm{R}}$ (minor) $5.7 \mathrm{~min}, t_{\mathrm{R}}$ (major) $7.5 \mathrm{~min}$. TLC (10/90 EtOAc/ hexanes): $R_{f}=0.47$.

$[\alpha]_{\mathrm{D}}^{25}=-53.0\left(c=2.0, \mathrm{CHCl}_{3}\right)$.

\section{Di-tert-butyl (S)-2-(2-(4-Fluorophenyl)-1-(isopropylthio)-2-oxoethyl)malonate}

(30)-The title compound was prepared according to the general procedure using propane-2-thiol ( $0.05 \mathrm{~mL}, 0.54 \mathrm{mmol}, 5.38$ equiv). White solid $(39.2 \mathrm{mg}, 0.093 \mathrm{mmol}$, 93\%), mp 71-73 ${ }^{\circ} \mathrm{C}$ (decomp); ${ }^{1} \mathrm{H}$ NMR (600 MHz, $\mathrm{CDCl}_{3}$ ) $\delta 8.10-8.07$ (m, 2H), 7.17 (app $\mathrm{t}, J=8.6 \mathrm{~Hz}, 2 \mathrm{H}), 4.71(\mathrm{~d}, J=11.6 \mathrm{~Hz}, 1 \mathrm{H}), 4.16(\mathrm{~d}, J=11.5 \mathrm{~Hz}, 1 \mathrm{H}), 2.95-2.88(\mathrm{~m}, 1 \mathrm{H})$, 1.55 (s, 9H), 1.39 (s, 9H), 1.24 (d, $J=6.8 \mathrm{~Hz}, 3 \mathrm{H}), 1.09$ (d, $J=6.8 \mathrm{~Hz}, 3 \mathrm{H}) ;{ }^{13} \mathrm{C}$ NMR $(151$ $\left.\mathrm{MHz}, \mathrm{CDCl}_{3}\right) \delta 193.3,167.0,166.8,165.7(\mathrm{~d}, J=254.7 \mathrm{~Hz}), 132.2(\mathrm{~d}, J=3.0 \mathrm{~Hz}), 131.3(\mathrm{~d}$, $J=9.2 \mathrm{~Hz}), 115.7(\mathrm{~d}, J=21.9 \mathrm{~Hz}), 82.5,82.2,55.7,45.7,34.6,27.9,27.8,24.8,24.2 ;{ }^{19} \mathrm{~F}$ NMR $\left(376 \mathrm{MHz}, \mathrm{CDCl}_{3}\right) \delta-105.41$. IR (thin film) $v 3431,2979,2360,1728,1678,1598$, $1369,1308,1157,862 \mathrm{~cm}^{-1}$. HRMS (ESI): Calcd For $\mathrm{C}_{22} \mathrm{H}_{31} \mathrm{FO}_{5} \mathrm{SNa}^{+}\left(\left[\mathrm{M}+\mathrm{Na}^{+}\right]\right)$: 449.1774, found 449.1764. HPLC Chiralpak IC column, $\mathrm{Hex} /{ }^{i} \mathrm{PrOH}=98: 2$, flow rate $=1.0$ $\mathrm{mL} / \mathrm{min}, \lambda=210 \mathrm{~nm}, t_{\mathrm{R}}$ (minor) $5.6 \mathrm{~min}, t_{\mathrm{R}}$ (major) $6.8 \mathrm{~min}$. TLC (10/90 EtOAc/hexanes): $R_{f}=0.43 .[\alpha]_{\mathrm{D}}^{25}=-70.0\left(c=2.0, \mathrm{CHCl}_{3}\right)$.

\section{Di-tert-butyl (S)-2-(1-(Isopropylthio)-2-(4-nitrophenyl)-2-oxoethyl)malonate (3p)}

-The title compound was prepared according to the general procedure using propane-2thiol (0.05 mL, $0.54 \mathrm{mmol}, 5.38$ equiv). Clear oil ( $42.0 \mathrm{mg}, 0.093 \mathrm{mmol}, 93 \%) ;{ }^{1} \mathrm{H}$ NMR $\left(600 \mathrm{MHz}, \mathrm{CDCl}_{3}\right) \delta 8.34(\mathrm{~d}, J=8.9 \mathrm{~Hz}, 2 \mathrm{H}), 8.20(\mathrm{~d}, J=8.9 \mathrm{~Hz}, 2 \mathrm{H}), 4.70(\mathrm{~d}, J=11.5 \mathrm{~Hz}$, $1 \mathrm{H}), 4.16(\mathrm{~d}, J=11.5 \mathrm{~Hz}, 1 \mathrm{H}), 2.94-2.87(\mathrm{~m}, 1 \mathrm{H}), 1.56(\mathrm{~s}, 9 \mathrm{H}), 1.41$ (s, 9H), 1.24 (d, $J=6.8$ $\mathrm{Hz}, 3 \mathrm{H}), 1.10(\mathrm{~d}, J=6.8 \mathrm{~Hz}, 3 \mathrm{H}) ;{ }^{13} \mathrm{C} \mathrm{NMR}\left(151 \mathrm{MHz}, \mathrm{CDCl}_{3}\right) \delta 193.1,167.1,166.6$, $150.2,141.1,129.7,123.8,82.9,82.5,55.6,46.4,34.9,27.9,27.8,24.8,24.1$. IR (thin film) $v 3439,2978,2931,1738,1685,1530,1307,1139,850,702 \mathrm{~cm}^{-1}$. HRMS (ESI): Calcd For $\mathrm{C}_{22} \mathrm{H}_{31} \mathrm{NO}_{7} \mathrm{SNa}^{+}\left(\left[\mathrm{M}+\mathrm{Na}^{+}\right]\right): 476.1719$, found 476.1712. HPLC Chiralpak IC column, $\mathrm{Hex} / \mathrm{i} \mathrm{PrOH}=98: 2$, flow rate $=1.0 \mathrm{~mL} / \mathrm{min}, \lambda=210 \mathrm{~nm}, t_{\mathrm{R}}$ (minor) $9.6 \mathrm{~min}, t_{\mathrm{R}}$ (major) 13.0 $\min$. TLC (10/90 EtOAc/hexanes): $R_{f}=0.39 .[\alpha]_{\mathrm{D}}^{25}=-46.6\left(c=2.0, \mathrm{CHCl}_{3}\right)$.

\section{Di-tert-butyl (S)-2-(2-(4-Cyanophenyl)-1-(isopropylthio)-2-oxoethyl)malonate}

$(3 q)$-The title compound was prepared according to the general procedure using 
propane-2-thiol ( $0.05 \mathrm{~mL}, 0.54 \mathrm{mmol}, 5.38$ equiv). The crude material was purified via flash column chromatography using a gradient from 97.5/2.5 hexanes/EtOAc to 95/5 hexanes/ EtOAc. White solid (38.8 mg, $0.091 \mathrm{mmol}, 91 \%), \mathrm{mp} 83-85{ }^{\circ} \mathrm{C}$ (decomp); ${ }^{1} \mathrm{H}$ NMR (600 $\left.\mathrm{MHz}, \mathrm{CDCl}_{3}\right) \delta 8.13(\mathrm{~d}, J=8.4 \mathrm{~Hz}, 2 \mathrm{H}), 7.80(\mathrm{~d}, J=8.5 \mathrm{~Hz}, 2 \mathrm{H}), 4.67(\mathrm{~d}, J=11.6 \mathrm{~Hz}, 1 \mathrm{H})$, $4.15(\mathrm{~d}, J=11.6 \mathrm{~Hz}, 1 \mathrm{H}), 2.92-2.86(\mathrm{~m}, 1 \mathrm{H}), 1.55(\mathrm{~s}, 9 \mathrm{H}), 1.40$ (s, 9H), 1.23 (d, $J=6.8 \mathrm{~Hz}$, $3 \mathrm{H}), 1.09(\mathrm{~d}, J=6.8 \mathrm{~Hz}, 3 \mathrm{H}) ;{ }^{13} \mathrm{C}$ NMR $\left(151 \mathrm{MHz}, \mathrm{CDCl}_{3}\right) \delta 193.3,167.0,166.6,139.4$, 132.4, 129.1, 118.0, 116.1, 82.8, 82.5, 55.6, 46.1, 34.8, 27.9, 27.8, 24.8, 24.1. IR (thin film) $v 2978,2231,1727,1685,1369,1298,1162,1140,866,755 \mathrm{~cm}^{-1}$. HRMS (ESI): Calcd For $\mathrm{C}_{23} \mathrm{H}_{31} \mathrm{NO}_{5} \mathrm{SNa}^{+}\left(\left[\mathrm{M}+\mathrm{Na}^{+}\right]\right): 456.1821$, found 456.1815. HPLC Chiralpak IC column, $\mathrm{Hex} / \mathrm{PrOH}=96: 4$, flow rate $=1.0 \mathrm{~mL} / \mathrm{min}, \lambda=210 \mathrm{~nm}, t_{\mathrm{R}}$ (minor) $11.0 \mathrm{~min}, t_{\mathrm{R}}$ (major) $12.9 \mathrm{~min}$. TLC (10/90 EtOAc/hexanes): $R_{f}=0.30 .[\alpha]_{\mathrm{D}}^{25}=-51.0\left(c=2.0, \mathrm{CHCl}_{3}\right)$.

Di-tert-butyl (S)-2-(2-Cyclopropyl-1-(isopropylthio)-2-oxoethyl)-malonate (3r)The title compound was prepared according to the general procedure using propane-2-thiol (0.05 mL, $0.54 \mathrm{mmol}, 5.38$ equiv). Low-melting white solid (33.2 $\mathrm{mg}, 0.092 \mathrm{mmol}, 92 \%$ ); ${ }^{1} \mathrm{H}$ NMR $\left(600 \mathrm{MHz}, \mathrm{CDCl}_{3}\right) \delta 4.07(\mathrm{~d}, J=11.6 \mathrm{~Hz}, 1 \mathrm{H}), 3.88(\mathrm{~d}, J=11.6 \mathrm{~Hz}, 1 \mathrm{H}), 2.98-$ $2.91(\mathrm{~m}, 1 \mathrm{H}), 2.26-2.22(\mathrm{~m}, 1 \mathrm{H}), 1.51(\mathrm{~s}, 9 \mathrm{H}), 1.43(\mathrm{~s}, 9 \mathrm{H}), 1.31(\mathrm{~d}, J=6.8 \mathrm{~Hz}, 3 \mathrm{H}), 1.22$ (d, $J=6.8 \mathrm{~Hz}, 3 \mathrm{H}), 1.13-1.09(\mathrm{~m}, 1 \mathrm{H}), 1.09-1.05(\mathrm{~m}, 1 \mathrm{H}), 1.01-0.96(\mathrm{~m}, 2 \mathrm{H}) ;{ }^{13} \mathrm{C}$ NMR $\left(151 \mathrm{MHz}, \mathrm{CDCl}_{3}\right) \delta 204.7,166.7,166.7,82.2,82.0,55.3,51.2,34.8,27.9,27.8,24.8,24.1$, 19.5, 12.1, 11.2. IR (thin film) $v 3445,2979,2931,1732,1609,1369,1255,1141,1054$, $851 \mathrm{~cm}^{-1}$. HRMS (ESI): Calcd For $\mathrm{C}_{19} \mathrm{H}_{32} \mathrm{O}_{5} \mathrm{SNa}^{+}\left(\left[\mathrm{M}+\mathrm{Na}^{+}\right]\right): 395.1868$, found 395.1858 . HPLC Chiralpak AD column, $\mathrm{Hex} /{ }^{i} \mathrm{PrOH}=98: 2$, flow rate $=1.0 \mathrm{~mL} / \mathrm{min}, \lambda=210 \mathrm{~nm}, t_{\mathrm{R}}$ (major) $5.0 \mathrm{~min}, t_{\mathrm{R}}$ (minor) $10.5 \mathrm{~min}$. TLC (10/90 EtOAc/hexanes): $R_{f}=0.40$.

$[\alpha]_{\mathrm{D}}^{25}=-103.6\left(c=1.5, \mathrm{CHCl}_{3}\right)$.

Di-tert-butyl (S)-2-(2-(Furan-2-yl)-1-(isopropylthio)-2-oxoethyl)-malonate (3s)The title compound was prepared according to the general procedure using propane-2-thiol ( $0.05 \mathrm{~mL}, 0.54 \mathrm{mmol}, 5.38$ equiv). The crude material was purified via flash column chromatography using a gradient from 97.5/2.5 hexanes/EtOAc to 95/5 hexanes/EtOAc. White solid (36.9 mg, $0.091 \mathrm{mmol}, 91 \%)$, mp $103-105{ }^{\circ} \mathrm{C}$ (decomp); ${ }^{1} \mathrm{H} \mathrm{NMR}(600 \mathrm{MHz}$, $\left.\mathrm{CDCl}_{3}\right) \delta 7.62(\mathrm{~d}, J=0.9 \mathrm{~Hz}, 1 \mathrm{H}), 7.32(\mathrm{~d}, J=3.5 \mathrm{~Hz}, 1 \mathrm{H}), 6.58(\mathrm{dd}, J=3.5 \mathrm{~Hz}, 1.6 \mathrm{~Hz}$, $1 \mathrm{H}), 4.59$ (d, $J=11.8 \mathrm{~Hz}, 1 \mathrm{H}), 4.11(\mathrm{~d}, J=11.8 \mathrm{~Hz}, 1 \mathrm{H}), 3.10-3.03(\mathrm{~m}, 1 \mathrm{H}), 1.54$ (s, 9H), $1.38(\mathrm{~s}, 9 \mathrm{H}), 1.26(\mathrm{~d}, J=6.8 \mathrm{~Hz}, 3 \mathrm{H}), 1.19(\mathrm{~d}, J=6.7 \mathrm{~Hz}, 3 \mathrm{H}) ;{ }^{13} \mathrm{C} \mathrm{NMR}(151 \mathrm{MHz}$, $\left.\mathrm{CDCl}_{3}\right) \delta 183.9,166.8,166.7,151.3,146.3,118.0,112.6,82.5,82.2,55.3,45.8,35.1,27.9$, 27.8, 24.4, 24.0. IR (thin film) v3430, 2979, 2360, 1737, 1659, 1468, 1366, 1311, 1165, $1138 \mathrm{~cm}^{-1}$. HRMS (ESI): Calcd For $\mathrm{C}_{20} \mathrm{H}_{30} \mathrm{O}_{6} \mathrm{SNa}^{+}\left(\left[\mathrm{M}+\mathrm{Na}^{+}\right]\right): 421.1661$, found 421.1650. HPLC Chiralpak IC column, $\mathrm{Hex} /{ }^{i} \mathrm{PrOH}=96: 4$, flow rate $=1.0 \mathrm{~mL} / \mathrm{min}, \lambda=210$ $\mathrm{nm}, t_{\mathrm{R}}$ (minor) $12.1 \mathrm{~min}, t_{\mathrm{R}}$ (major) $17.5 \mathrm{~min}$. TLC (10/90 EtOAc/hexanes): $R_{f}=0.27$. $[\alpha]_{\mathrm{D}}^{25}=-56.2\left(c=2.0, \mathrm{CHCl}_{3}\right)$.

Di-tert-butyl (S)-2-(2-(Furan-2-yl)-1-(isopropylthio)-2-oxoethyl)-malonate (3t)The title compound was prepared according to the general procedure using propane-2-thiol ( $0.05 \mathrm{~mL}, 0.54 \mathrm{mmol}, 5.38$ equiv). The crude material was purified via flash column chromatography using a gradient from 97.5/2.5 hexanes/EtOAc to 95/5 hexanes/EtOAc. 
White solid (39.5 mg, $0.095 \mathrm{mmol}, 95 \%)$, mp 71-73 ${ }^{\circ} \mathrm{C}$ (decomp); ${ }^{1} \mathrm{H}$ NMR (600 MHz, $\left.\mathrm{CDCl}_{3}\right) \delta 7.86(\mathrm{dd}, J=3.8 \mathrm{~Hz}, 1.1 \mathrm{~Hz}, 1 \mathrm{H}), 7.67(\mathrm{dd}, J=4.9 \mathrm{~Hz}, 1.0 \mathrm{~Hz}, 1 \mathrm{H}), 7.17(\mathrm{dd}, J=$ $4.9 \mathrm{~Hz}, 3.8 \mathrm{~Hz}, 1 \mathrm{H}), 4.58(\mathrm{~d}, J=11.6 \mathrm{~Hz}, 1 \mathrm{H}), 4.13(\mathrm{~d}, J=11.6 \mathrm{~Hz}, 1 \mathrm{H}), 3.08-2.99(\mathrm{~m}, 1 \mathrm{H})$, $1.55(\mathrm{~s}, 9 \mathrm{H}), 1.38(\mathrm{~s}, 9 \mathrm{H}), 1.27(\mathrm{~d}, J=6.8 \mathrm{~Hz}, 3 \mathrm{H}), 1.16(\mathrm{~d}, J=6.8 \mathrm{~Hz}, 3 \mathrm{H}) ;{ }^{13} \mathrm{C} \mathrm{NMR}(151$ $\left.\mathrm{MHz}, \mathrm{CDCl}_{3}\right) \delta 187.9,166.8,166.7,142.4,133.8,132.4,128.2,82.5,82.2,55.7,47.1,34.7$, 27.9, 27.8, 24.7, 24.2. IR (thin film) v 3431, 2978, 1730, 1659, 1415, 1369, 1304, 1161, $849,724 \mathrm{~cm}^{-1}$. HRMS (ESI): Calcd For $\mathrm{C}_{20} \mathrm{H}_{30} \mathrm{O}_{5} \mathrm{~S}_{2} \mathrm{Na}^{+}\left(\left[\mathrm{M}+\mathrm{Na}^{+}\right]\right)$: 437.1433, found 437.1423. HPLC Chiralpak IC column, $\mathrm{Hex} /{ }^{i} \mathrm{PrOH}=98: 2$, flow rate $=1.0 \mathrm{~mL} / \mathrm{min}, \lambda=210$ $\mathrm{nm}, t_{\mathrm{R}}$ (minor) $10.7 \mathrm{~min}, t_{\mathrm{R}}$ (major) $15.7 \mathrm{~min}$. TLC (10/90 EtOAc/hexanes): $R_{f}=0.33$. $[\alpha]_{\mathrm{D}}^{25}=-60.5\left(c=2.0, \mathrm{CHCl}_{3}\right)$.

\section{Di-tert-butyl (S)-2-(1-(Isopropylthio)-2-oxo-2-(pyridin-4-yl)ethyl)-malonate (3u)}

-The title compound was prepared according to the general procedure using propane-2thiol $(0.05 \mathrm{~mL}, 0.54 \mathrm{mmol}, 5.38$ equiv). The crude material was purified via flash column chromatography using a gradient from 90/10 hexanes/EtOAc to $85 / 15$ hexanes/EtOAc. White solid (37.3 mg, $0.090 \mathrm{mmol}, 90 \%)$, mp 69-71 ${ }^{\circ} \mathrm{C}$ (decomp); ${ }^{1} \mathrm{H}$ NMR (600 MHz, $\left.\mathrm{CDCl}_{3}\right) \delta 8.82(\mathrm{~d}, J=5.9 \mathrm{~Hz}, 2 \mathrm{H}), 7.82(\mathrm{dd}, J=4.4 \mathrm{~Hz}, 1.6 \mathrm{~Hz}, 2 \mathrm{H}), 4.64(\mathrm{~d}, J=11.6 \mathrm{~Hz}$, $1 \mathrm{H}), 4.13(\mathrm{~d}, J=11.6 \mathrm{~Hz}, 1 \mathrm{H}), 2.92-2.85(\mathrm{~m}, 1 \mathrm{H}), 1.54$ (s, 9H), 1.40 (s, 9H), 1.23 (d, $J=6.8$ $\mathrm{Hz}, 3 \mathrm{H}), 1.10(\mathrm{~d}, J=6.8 \mathrm{~Hz}, 3 \mathrm{H}) ;{ }^{13} \mathrm{C}$ NMR $\left(151 \mathrm{MHz}, \mathrm{CDCl}_{3}\right) \delta 193.8,167.0,166.5$, $150.8,142.5,121.7,82.9,82.5,55.4,46.1,34.9,27.9,27.8,24.7,24.1$. IR (thin film) $v$ 3431, 2979, 2360, 1728, 1691, 1369, 1301, 1141, 861, $667 \mathrm{~cm}^{-1}$. HRMS (ESI): Calcd For $\mathrm{C}_{21} \mathrm{H}_{32} \mathrm{NO}_{5} \mathrm{~S}^{+}\left(\left[\mathrm{M}+\mathrm{H}^{+}\right]\right): 410.2002$, found 410.1990. HPLC Chiralpak IA column, Hex/ ${ }^{i} \mathrm{PrOH}=98: 2$, flow rate $=1.0 \mathrm{~mL} / \mathrm{min}, \lambda=225 \mathrm{~nm}, t_{\mathrm{R}}$ (major) $7.2 \mathrm{~min}, t_{\mathrm{R}}$ (minor) $8.5 \mathrm{~min}$.

TLC (25/75 EtOAc/hexanes): $R_{f}=0.32 .[\alpha]_{\mathrm{D}}^{25}=-65.5\left(c=2.0, \mathrm{CHCl}_{3}\right)$.

\section{General Procedure for the Reduction of 3}

A vial under a $\mathrm{N}_{2}$ atmosphere was charged with $a$-sulfaketone $\mathbf{3}$ ( $0.15 \mathrm{mmol}, 1.0$ equiv), followed by $\mathrm{MeOH}(1.5 \mathrm{~mL})$. The reaction mixture was cooled to $-20{ }^{\circ} \mathrm{C}, \mathrm{NaBH}_{4}(10.3 \mathrm{mg}$, 1.75 equiv) was added, and stirring was continued at $-20^{\circ} \mathrm{C}$. After quenching with $1 \mathrm{M} \mathrm{HCl}$ $(1.5 \mathrm{~mL})$, the product was extracted with $\mathrm{DCM}(3 \times)$, dried with $\mathrm{Na}_{2} \mathrm{SO}_{4}$, and concentrated in vacuo. The crude material was purified via column chromatography using a gradient from 97.5/2.5 hexanes/EtOAc to $95 / 5$ hexanes/EtOAc.

\section{Di-tert-butyl 2-((1S,2S)-2-Hydroxy-1-(isopropylthio)-2-phenylethyl)malonate}

(4a)-The product was synthesized according to the general procedure, and quenched after $1 \mathrm{~h}$. White solid (53.7 mg, $0.131 \mathrm{mmol}, 87 \%$ ), mp 85-87 ${ }^{\circ} \mathrm{C}$ (decomp); ${ }^{1} \mathrm{H} \mathrm{NMR} \mathrm{(600} \mathrm{MHz,}$ $\left.\mathrm{CDCl}_{3}\right) \delta 7.46(\mathrm{~d}, J=7.5 \mathrm{~Hz}, 2 \mathrm{H}), 7.37(\mathrm{t}, J=7.6 \mathrm{~Hz}, 2 \mathrm{H}), 7.29(\mathrm{t}, J=7.3 \mathrm{~Hz}, 1 \mathrm{H}), 4.89$ (d, $J=3.4 \mathrm{~Hz}, 1 \mathrm{H}), 3.54-3.49(\mathrm{~m}, 2 \mathrm{H}), 2.39-2.32(\mathrm{~m}, 1 \mathrm{H}), 1.52(\mathrm{~s}, 9 \mathrm{H}), 1.51(\mathrm{~s}, 9 \mathrm{H}), 1.11$ (d, $J$ $=6.7 \mathrm{~Hz}, 3 \mathrm{H}), 0.95(\mathrm{~d}, J=6.7 \mathrm{~Hz}, 3 \mathrm{H}) ;{ }^{13} \mathrm{C} \mathrm{NMR}\left(151 \mathrm{MHz}, \mathrm{CDCl}_{3}\right) \delta 167.7,167.0,142.1$, 128.2, 127.6, 126.1, 82.6, 82.0, 76.8, 73.0, 57.2, 53.5, 37.4, 28.0, 23.3 (2C). IR (thin film) $v$ $3434,2979,2089,1725,1645,1454,1628,1251,1155,1139 \mathrm{~cm}^{-1}$. HRMS (ESI): Calcd For $\mathrm{C}_{22} \mathrm{H}_{34} \mathrm{O}_{5} \mathrm{SNa}^{+}\left(\left[\mathrm{M}+\mathrm{Na}^{+}\right]\right): 433.2025$, found 433.2012. HPLC Chiralpak IA column, Hex/ ${ }^{i} \mathrm{PrOH}=97: 3$, flow rate $=1.0 \mathrm{~mL} / \mathrm{min}, \lambda=210 \mathrm{~nm}, t_{\mathrm{R}}$ (major) $7.3 \mathrm{~min}, t_{\mathrm{R}}$ (minor) $8.8 \mathrm{~min}$.

TLC (10/90 EtOAc/hexanes): $R_{f}=0.29 .[\alpha]_{\mathrm{D}}^{25}=-7.33\left(c=0.75, \mathrm{CHCl}_{3}\right)$. 
Di-tert-butyl 2-((1S,2S)-1-(Benzylthio)-2-hydroxy-2-phenylethyl)-malonate (4f)The product was synthesized according to the general procedure, and quenched after $3 \mathrm{~h}$. White solid (49.6 mg, $0.108 \mathrm{mmol}, 74 \%)$, mp 81-83 ${ }^{\circ} \mathrm{C}$ (decomp); ${ }^{1} \mathrm{H}$ NMR (600 MHz, $\left.\mathrm{CDCl}_{3}\right) \delta 7.43(\mathrm{~d}, J=7.4 \mathrm{~Hz}, 2 \mathrm{H}), 7.38(\mathrm{t}, J=7.6 \mathrm{~Hz}, 2 \mathrm{H}), 7.32(\mathrm{t}, J=7.3 \mathrm{~Hz}, 1 \mathrm{H}), 7.26-$ $7.20(\mathrm{~m}, 3 \mathrm{H}), 7.08(\mathrm{~d}, J=6.5 \mathrm{~Hz}, 2 \mathrm{H}), 4.94(\mathrm{~d}, J=1.9 \mathrm{~Hz}, 1 \mathrm{H}), 3.53-3.48(\mathrm{~m}, 3 \mathrm{H}), 3.44(\mathrm{br}$ s, $1 \mathrm{H}), 3.29(\mathrm{~d}, J=12.2 \mathrm{~Hz}, 1 \mathrm{H}), 1.52(\mathrm{~s}, 9 \mathrm{H}), 1.51(\mathrm{~s}, 9 \mathrm{H}) ;{ }^{13} \mathrm{C} \mathrm{NMR}\left(151 \mathrm{MHz}, \mathrm{CDCl}_{3}\right) \delta$ 167.7, 166.9, 141.9, 137.4, 129.0, 128.4, 128.3, 127.7, 127.2, 126.1, 82.7, 82.2, 73.0, 57.0, 54.3, 38.6, 28.0 (2C). IR (thin film) v 3459, 2979, 2359, 2341, 1731, 1455, 1369, 1254, 1140, $700 \mathrm{~cm}^{-1}$. HRMS (ESI): Calcd For $\mathrm{C}_{26} \mathrm{H}_{34} \mathrm{O}_{5} \mathrm{SNa}^{+}\left(\left[\mathrm{M}+\mathrm{Na}^{+}\right]\right)$: 481.2025 , found 481.2011. HPLC Chiralpak IA column, $\mathrm{Hex} / \mathrm{PrOH}=97: 3$, flow rate $=1.0 \mathrm{~mL} / \mathrm{min}, \lambda=210$ $\mathrm{nm}, t_{\mathrm{R}}$ (major) $10.2 \mathrm{~min}, t_{\mathrm{R}}$ (minor) $14.4 \mathrm{~min}$. TLC (10/90 EtOAc/hexanes): $R_{f}=0.27$. $[\alpha]_{\mathrm{D}}^{25}=+18.4\left(c=2.0, \mathrm{CHCl}_{3}\right)$.

\section{tert-Butyl (3S,4S,5S)-4-(Isopropylthio)-2-oxo-5-phenyltetrahydro-furan-3-} carboxylate (5a)-A vial under an atmosphere of $\mathrm{N}_{2}$ was charged with $\mathbf{4 a}(41.3 \mathrm{mg}, 0.10$ $\mathrm{mmol}, 1.0$ equiv) and 1,4-dioxane $(0.1 \mathrm{M})$. Then, $4 \mathrm{M} \mathrm{HCl}$ in 1,4-dioxane $(0.1 \mathrm{M})$ was added dropwise at room temperature. The reaction was stirred for $5 \mathrm{~h}$, then diluted with $\mathrm{Et}_{2} \mathrm{O}$ and $\mathrm{H}_{2} \mathrm{O}$. The layers were separated, and the aqueous layer was extracted with $\mathrm{Et}_{2} \mathrm{O}$. The combined organic layers were dried with $\mathrm{Na}_{2} \mathrm{SO}_{4}$ and concentrated in vacuo. The crude material was then purified with flash column chromatography using $50 / 50 \mathrm{DCM} /$ hexanes to give an off-white solid (25.5 mg, $0.075 \mathrm{mmol}, 75 \%$ ), mp 70-72 ${ }^{\circ} \mathrm{C}$ (decomp); ${ }^{1} \mathrm{H}$ NMR (600 $\left.\mathrm{MHz}, \mathrm{CDCl}_{3}\right) \delta 7.43-7.39(\mathrm{~m}, 3 \mathrm{H}), 7.29-7.28(\mathrm{~m}, 2 \mathrm{H}), 5.81(\mathrm{~d}, J=7.4 \mathrm{~Hz}, 1 \mathrm{H}), 4.32(\mathrm{t}, J=$ $7.7 \mathrm{~Hz}, 1 \mathrm{H}), 3.68(\mathrm{~d}, J=7.9 \mathrm{~Hz}, 1 \mathrm{H}), 2.74-2.68(\mathrm{~m}, 1 \mathrm{H}), 1.56(\mathrm{~s}, 9 \mathrm{H}), 1.22(\mathrm{~d}, J=6.6 \mathrm{~Hz}$, $3 \mathrm{H}), 1.18(\mathrm{~d}, J=6.8 \mathrm{~Hz}, 3 \mathrm{H}) ;{ }^{13} \mathrm{C}$ NMR $\left(151 \mathrm{MHz}, \mathrm{CDCl}_{3}\right) \delta 170.9,165.9,134.9,129.0$, $128.3,126.6,83.7,82.5,55.5,46.8,35.5,27.9,23.5,23.2$. IR (thin film) $v 3436,1783$, 1732, 1647, 1636, 1456, 1135, 1271, 1034, $978 \mathrm{~cm}^{-1}$. HRMS (ESI): Calcd For $\mathrm{C}_{18} \mathrm{H}_{24} \mathrm{O}_{4} \mathrm{SNa}^{+}\left(\left[\mathrm{M}+\mathrm{Na}^{+}\right]\right): 359.1293$, found 359.1285. HPLC Chiralpak IC column, Hex/ ${ }^{i} \mathrm{PrOH}=96: 4$, flow rate $=1.0 \mathrm{~mL} / \mathrm{min}, \lambda=210 \mathrm{~nm}, t_{\mathrm{R}}$ (minor) $11.4 \mathrm{~min}, t_{\mathrm{R}}$ (major) 12.6 $\min$. TLC (10/90 EtOAc/hexanes): $R_{f}=0.34 .[\alpha]_{\mathrm{D}}^{25}=-39.8\left(c=1.0, \mathrm{CHCl}_{3}\right)$.

\section{tert-Butyl (3S,4S,5S)-4-(Benzylthio)-2-oxo-5-phenyltetrahydrofuran-3-}

carboxylate (5f)—A vial under an atmosphere of $\mathrm{N}_{2}$ was charged with $\mathbf{4 f}(41.3 \mathrm{mg}, 0.10$ mmol, 1.0 equiv) and $4 \mathrm{M} \mathrm{HCl}$ in 1,4-dioxane $(2 \mathrm{~mL})$. The reaction was stirred for $2 \mathrm{~h}$, then diluted with $\mathrm{Et}_{2} \mathrm{O}$ and $\mathrm{H}_{2} \mathrm{O}$. The layers were separated, and the aqueous layer was extracted with $\mathrm{Et}_{2} \mathrm{O}$. The combined organic layers were dried with $\mathrm{Na}_{2} \mathrm{SO}_{4}$ and concentrated in vacuo. The crude material was then purified with flash column chromatography using 50/50 DCM/ hexanes to give an off-white solid (25.5 mg, $0.067 \mathrm{mmol}, 67 \%), \mathrm{mp} 94-96{ }^{\circ} \mathrm{C}$ (decomp); ${ }^{1} \mathrm{H}$ NMR (600 MHz, CDCl $)_{3} \delta 7.43-7.41(\mathrm{~m}, 3 \mathrm{H}), 7.33(\mathrm{t}, J=7.2 \mathrm{~Hz}, 2 \mathrm{H}), 7.29-7.27(\mathrm{~m}, 3 \mathrm{H})$, $7.21(\mathrm{~d}, J=7.0 \mathrm{~Hz}, 2 \mathrm{H}), 5.73(\mathrm{~d}, J=7.0 \mathrm{~Hz}, 1 \mathrm{H}), 4.11(\mathrm{t}, J=6.8 \mathrm{~Hz}, 1 \mathrm{H}), 3.69(\mathrm{~d}, J=6.7$ $\mathrm{Hz}, 1 \mathrm{H}), 3.49$ (d, $J=13.1 \mathrm{~Hz}, 1 \mathrm{H}), 3.45(\mathrm{~d}, J=13.1 \mathrm{~Hz}, 1 \mathrm{H}), 1.55(\mathrm{~s}, 9 \mathrm{H}) ;{ }^{13} \mathrm{C}$ NMR $(151$ $\left.\mathrm{MHz}, \mathrm{CDCl}_{3}\right) \delta 170.6,165.6,136.8,134.7,129.1,128.9,128.7,128.3,127.5,126.5,83.8$, 82.3, 55.3, 47.8, 36.2, 27.9. IR (thin film) $v 3437,1783,1731,1636,1456,1370,1138$, 1004, 979, $699 \mathrm{~cm}^{-1}$. HRMS (ESI): Calcd For $\mathrm{C}_{22} \mathrm{H}_{24} \mathrm{O}_{4} \mathrm{SNa}^{+}\left(\left[\mathrm{M}+\mathrm{Na}^{+}\right]\right)$: 407.1293, found 407.1286. HPLC Chiralpak IC column, $\mathrm{Hex} / \mathrm{PPOH}=96: 4$, flow rate $=1.0 \mathrm{~mL} / \mathrm{min}, \lambda$ 
$=210 \mathrm{~nm}, t_{\mathrm{R}}$ (major) $16.0 \mathrm{~min}, t_{\mathrm{R}}$ (minor) $18.2 \mathrm{~min} . \mathrm{TLC}$ (10/90 EtOAc/hexanes): $R_{f}=$

0.29. $[\alpha]_{\mathrm{D}}^{25}=-49.3\left(c=1.0, \mathrm{CHCl}_{3}\right)$.

\section{Supplementary Material}

Refer to Web version on PubMed Central for supplementary material.

\section{Acknowledgments}

The work described was supported by Award R35 GM118055 from the National Institute of General Medical Sciences. J.L.F. is an NSF Graduate Research Fellow. E.L.B. acknowledges support from the NSF REU program (CHE-1460874). We thank Dr. Brandie Ehrmann and Evan McConnell (UNC Department of Chemistry Mass Spectrometry Core Laboratory) for their assistance with mass spectrometry analysis and Blane Zavesky (UNC Department of Chemistry X-ray Structure Facility) for X-ray crystallographic analysis.

\section{References}

1. (a) Jacob C. Nat Prod Rep. 2006; 23:851-863. [PubMed: 17119635] (b) Ondetti MA, Rubin B, Cushman DW. Science. 1977; 196:441-444. [PubMed: 191908] (c) Kim J, Ashenhurst JA, Movassaghi M. Science. 2009; 324:238-241. [PubMed: 19359584] (d) Nudelman, A. The Chemistry of Optically Active Sulfur Compounds. Gordon and Breach; New York: 1984. (e) Damani, LA., editor. Sulphur-Containing Drugs and Related Organic Compounds. Wiley; New York: 1989.

2. (a) Pellissier, H., editor. Chiral Sulfur Ligands: Asymmetric Catalysis. RSC Publishing; Cambridge, U.K: 2009. (b) Gauthier MA, Gibson MI, Klok HA. Angew Chem, Int Ed. 2009; 48:48-58.(c) Dondoni A. Angew Chem, Int Ed. 2008; 47:8995-8997.(d) Hoyle CE, Bowman CN. Angew Chem, Int Ed. 2010; 49:1540-1573.(e) Legros J, Dehli JR, Bolm C. Adv Synth Catal. 2005; 347:19-31.(f) Dénès F, Schiesser CH, Renaud P. Chem Soc Rev. 2013; 42:7900-7942. [PubMed: 23828205] (g) Liebeskind LS, Srogl J. Org Lett. 2002; 4:979-981. [PubMed: 11893201]

3. For reviews of sulfa-Michael additions: Enders D, Lüttgen K, Narine AA. Synthesis. 2007; 2007:959-980.Chauhan P, Mahajan S, Enders D. Chem Rev. 2014; 114:8807-8864. [PubMed: 25144663] For selected recent examples: Fang X, Wang CJ. Chem Commun. 2015; 51:11851197. Chatupheeraphat A, Liao HH, Mader S, Sako M, Sasai H, Atodiresei I, Rueping M. Angew Chem, Int Ed. 2016; 55:4803-4807.Guo W, Wu B, Zhou X, Chen P, Wang X, Zhou YG, Liu Y, Li C. Angew Chem, Int Ed. 2015; 54:4522-4526.

4. Kowalczyk R, Wierzba AJ, Boratyński PJ, Bąkowicz J. Tetrahedron. 2014; 70:5834-5842.For an example with acrylamide functionality instead of acrylate: Zhao F, Zhang W, Yang Y, Pan Y, Chen W, Liu H, Yan L, Tan CH, Jiang Z. Adv Synth Catal. 2011; 353:2624-2630.

5. Yang J, Farley AJM, Dixon DJ. Chem Sci. 2017; 8:606-610. [PubMed: 28451207]

6. For the development of this catalyst family and recent applications, see: Núñez MG, Farley AJM, Dixon DJ. J Am Chem Soc. 2013; 135:16348-116351. [PubMed: 24107070] Goldys AM, Dixon DJ. Macromolecules. 2014; 47:1277-1284.Goldys AM, Núñez MG, Dixon DJ. Org Lett. 2014; 16:6294-6297. [PubMed: 25459386] Farley AJM, Sandford C, Dixon DJ. J Am Chem Soc. 2015; 137:15992-15995. [PubMed: 26679772] Robertson GP, Farley AJM, Dixon DJ. Synlett. 2016; 27:21-24.Horwitz MA, Zavesky BP, Martinez-Alvarado JI, Johnson JS. Org Lett. 2016; 18:36-39. [PubMed: 26667068] Shi H, Michaelides IN, Darses B, Jakubec P, Nguyen QNN, Paton RS, Dixon DJ. J Am Chem Soc. 2017; 139:17755-17758. [PubMed: 29120635]

7. Horwitz MA, Fulton JL, Johnson JS. Org Lett. 2017; 19:5783-5785. [PubMed: 29035548]

8 . When a large excess ( 5 equiv) of substituted benzyl thiol was employed, a new side product was observed (the alkane resulting from reduction of the alkene in $\mathbf{1}$ ), and enantioselectivity decreased significantly. We hypothesize that the excess thiol racemizes the conjugate addition product via formation of the disulfide. The resulting enolate (or equivalent) can then either attack the disulfide to form the racemic product or become protonated to give the observed reduction product. Aryl thiols were unsuccessful in this reaction as racemization proved unavoidable. 
9. (a) Hoffmann RW. Synthesis. 2004; 2004:2075-2090.(b) Horwitz MA, Johnson JS. Eur J Org Chem. 2017; 2017:1381-1390.

10. (a) Kano S, Yokomatsu T, Shibuya S. J Chem Soc, Chem Commun. 1978; 0:785-786.(b) Shimagaki M, Maeda T, Matsuzaki Y, Hori I, Nakata T, Oishi T. Tetrahedron Lett. 1984; 25:47754778.

11. CCDC 1587179 contains the supplementary crystallographic data for this paper. These data can be obtained free of charge from the Cambridge Crystallographic Centre via www.ccdc.cam.ac.uk/ data_request/cif 


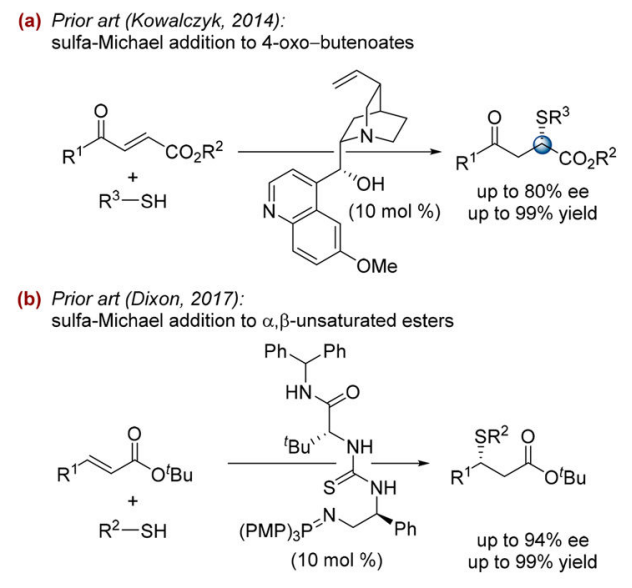

(c) Prior art (Johnson, 2017): conjugate addition of nitroalkanes to enone diesters

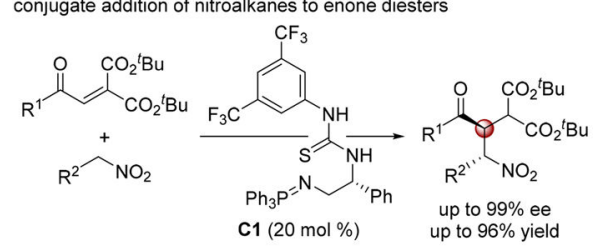

(d) Current work:

sulfa-Michael addition to functionalized enone diesters

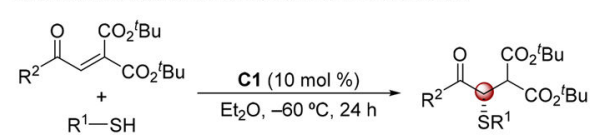

Scheme 1.

Asymmetric Conjugate Addition Reactions 
<smiles>CC(C)OC(=O)C(=CC(=O)c1ccccc1)C(=O)OC(C)(C)C</smiles>

(5.4 equiv)<smiles>FC(F)(F)c1cc(NC(=S)N[C@@H](CN=P)c2ccccc2)cc(C(F)(F)F)c1</smiles><smiles>COC(=O)C(C(=O)OCc1ccccc1)[C@H](SC(C)C)C(=O)c1ccccc1</smiles><smiles>CC(C=O)OC(C)([O])[O]</smiles>

$\mathrm{Et}_{2} \mathrm{O},-60^{\circ} \mathrm{C}$ $24 \mathrm{~h}$

1 a

$1.21 \mathrm{~g}$ $93 \%$ yield 97:3 er

Scheme 2.

Gram-Scale Asymmetric Sulfa-Michael Reaction 

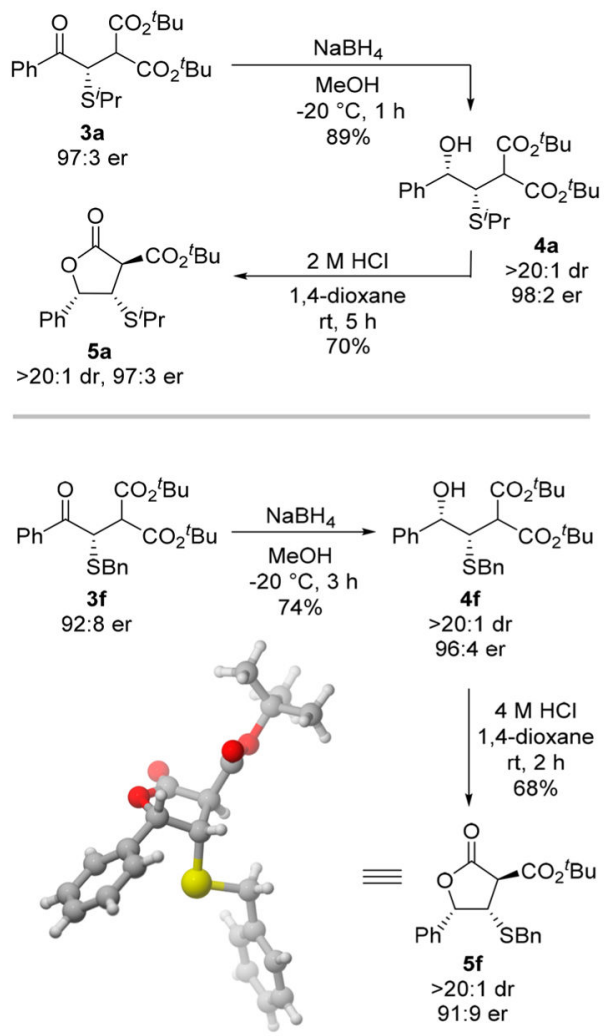

Scheme 3.

Local Desymmetrization via Diastereoselective Reduction and Lactonization 
Table 1

Asymmetric Conjugate Addition of Alkyl Thiols to Enone Diester 1a ${ }^{a}$

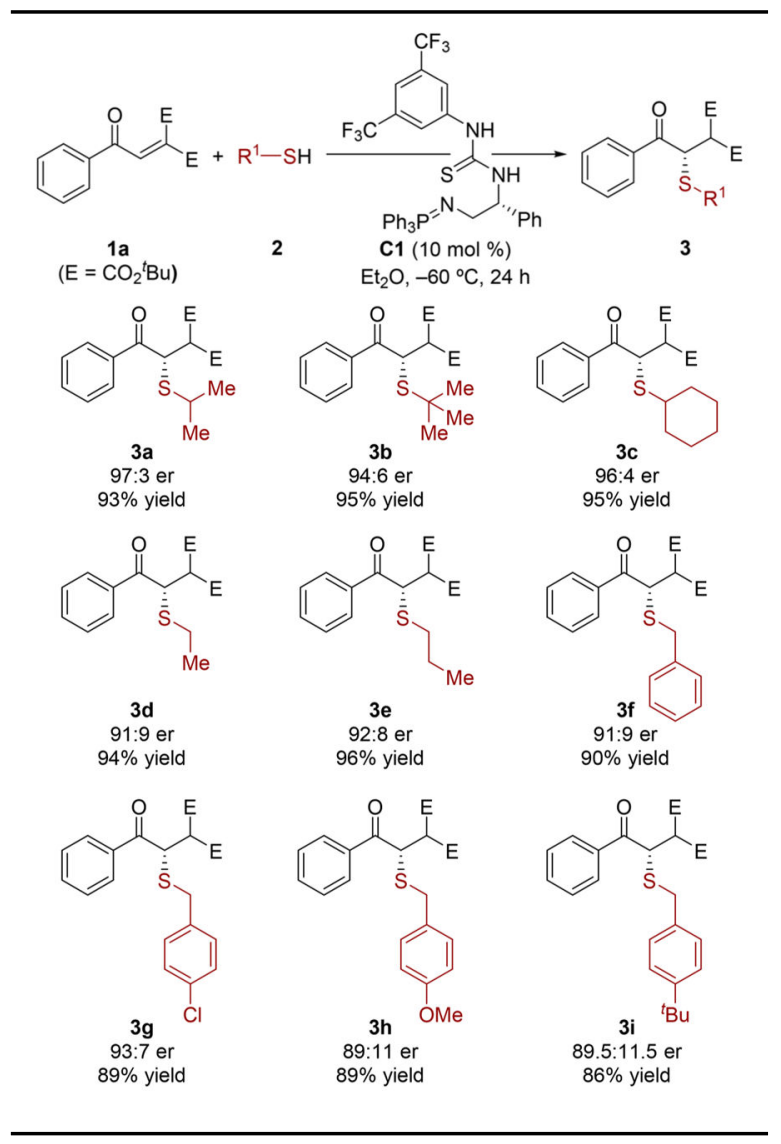

${ }^{a}$ All of the reactions were conducted on a $0.1 \mathrm{mmol}$ scale using 1.5-5.5 equiv of alkyl thiol 2 (see the Experimental Section for specific conditions). Isolated yields are shown. All yields and er values are the average of two trials. 
Table 2

Asymmetric Conjugate Addition of 2-Propanethiol to Enone Diester $1^{a}$

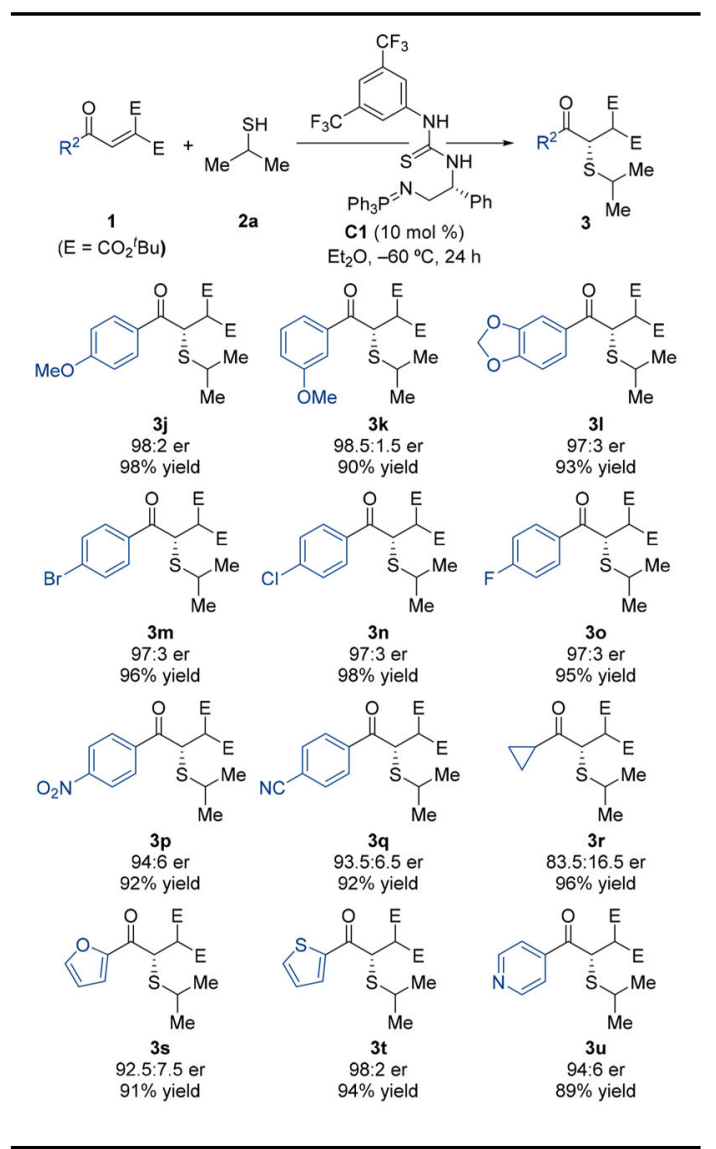

${ }^{a}$ All of the reactions were conducted on a 0.1 mmol scale using 5.4 equiv of alkyl thiol 2a. Isolated yields are shown. All yields and er values are the average of two trials. 